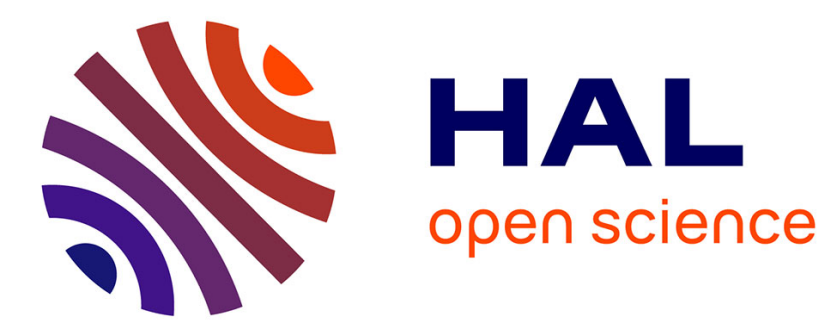

\title{
Long term evolution and chaotic diffusion of the insolation quantities of Mars.
}

\author{
Jacques Laskar, A.C.M. Correia, Mickael Gastineau, Frédéric Joutel,
} Benjamin Levrard, Philippe Robutel

\section{- To cite this version:}

Jacques Laskar, A.C.M. Correia, Mickael Gastineau, Frédéric Joutel, Benjamin Levrard, et al.. Long term evolution and chaotic diffusion of the insolation quantities of Mars.. 2004. hal-00000860

\section{HAL Id: hal-00000860 https://hal.science/hal-00000860}

Preprint submitted on 26 Mar 2004

HAL is a multi-disciplinary open access archive for the deposit and dissemination of scientific research documents, whether they are published or not. The documents may come from teaching and research institutions in France or abroad, or from public or private research centers.
L'archive ouverte pluridisciplinaire HAL, est destinée au dépôt et à la diffusion de documents scientifiques de niveau recherche, publiés ou non, émanant des établissements d'enseignement et de recherche français ou étrangers, des laboratoires publics ou privés. 


\title{
Long term evolution and chaotic diffusion of the insolation quantities of Mars.
}

\author{
J. Laskar ${ }^{a, *}$ A. C. M. Correia ${ }^{a, b, c}$ M. Gastineau ${ }^{a}$ F. Joutel,${ }^{a}$ B. Levrard,${ }^{a}$ and P. Robutel ${ }^{a}$ \\ ${ }^{a}$ Astronomie et Systèmes Dynamiques, IMCCE-CNRS UMR8028, 77 Av. Denfert-Rochereau, 75014 Paris, France \\ ${ }^{b}$ Observatoire de Genève, 51 chemin des Maillettes, 1290 Sauverny, Switzerland \\ ${ }^{c}$ Departamento de Física da Universidade de Aveiro, Campus Universitário de Santiago, 3810-193 Aveiro, Portugal
}

March 26, 2004

\section{Abstract}

As the obliquity of Mars is strongly chaotic, it is not possible to give a solution for its evolution over more than a few million years. Using the most recent data for the rotational state of Mars, and a new numerical integration of the Solar System, we provide here a precise solution for the evolution of Mars' spin over 10 to 20 Myr. Over $250 \mathrm{Myr}$, we present a statistical study of its possible evolution, when considering the uncertainties in the present rotational state. Over much longer time span, reaching 5 Gyr, chaotic diffusion prevails, and we have performed an extensive statistical analysis of the orbital and rotational evolution of Mars, relying on Laskar's secular solution of the Solar System, based on more than 600 orbital and 200000 obliquity solutions over 5 Gyr. The density functions of the eccentricity and obliquity are specified with simple analytical formulas. We found an averaged eccentricity of Mars over 5 Gyr of 0.0690 with standard deviation 0.0299 , while the averaged value of the obliquity is $37.62^{\circ}$ with a standard deviation of $13.82^{\circ}$, and a maximal value of $82.035^{\circ}$. We find that the probability for Mars' obliquity to have reached more than $60^{\circ}$ in the past 1 Gyr is $63.0 \%$, and $89.3 \%$ in 3 Gyr. Over 4 Gyr, the position of Mars' axis is given by a uniform distribution on a spherical cap limited by the obliquity $58.62^{\circ}$, with the addition of a random noise allowing a slow diffusion beyond this limit. We can also define a standard model of Mars' insolation parameters over $4 \mathrm{Gyr}$ with the most probable values 0.068 for the eccentricity and $41.80^{\circ}$ for the obliquity.

Keywords : Mars, Climate; Rotational Dynamics; Resonances; Planetary Dynamics

\section{Contents}

1 Introduction 1

2 Evolution over 250 Myr 3

2.1 Orbital Motion . . . . . . . . . . . . 3

2.2 Numerical integrator . . . . . . . . . . . . . . 3

2.3 Comparison with DE406 . . . . . . . . . . 4

2.4 Variations on the orbital model . . . . . . . 5

3 Precession equations $\quad 6$

3.1 Dissipative effects . . . . . . . . . . . . 6

3.1.1 Tidal dissipation . . . . . . . . . . 6

3.1.2 Core-mantle friction . . . . . . . . 7

3.1.3 Climate friction . . . . . . . . . . . 8

3.2 Initial spin axis orientation $\ldots \ldots \ldots .8$

3.2.1 Precession constant . . . . . . . . 9 9

3.2.2 The solution La2004 . . . . . . . . 10

4 Variations over 250 Ma 12

4.1 Maximum variations over $250 \mathrm{Ma}$. . . . . . 12

4.2 The chaotic zone . . . . . . . . . . . 12

4.3 Obliquity statistics over $250 \mathrm{Myr} \ldots \ldots . .13$

5 Diffusion over 5 Gyr $\quad 14$

5.1 The secular equations . . . . . . . . . 14

5.2 Eccentricity . . . . . . . . . . . . . . 15

5.3 Obliquity . . . . . . . . . . . . . 16

5.4 Diffusion laws . . . . . . . . . . . . . . . 17

5.4.1 Eccentricity . . . . . . . . . . 18

5.4 .2 Obliquity . . . . . . . . . . . 19

6 Conclusions 21

${ }^{1}$ E-mail address: laskar@imcce.fr

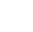

(1)

(1)

5

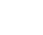

6

6

7

8

8

9

10

2

12

2




\section{Introduction}

The parameters of Mars' orbit and spin axis orientation control the global distribution and seasonal intensity of the solar insolation, and it is widely accepted that astronomical variations could have had a profound influence on its climatic history. These changes are probably characterized by a redistribution of the major Martian volatiles $\left(\mathrm{CO}_{2}\right.$, dust and water $)$ and variations in their partition between atmospheric, surface, and subsurface reservoirs.

Because the permanent $\mathrm{CO}_{2}$ cap is in equilibrium with the atmosphere, the Martian atmospheric pressure is very sensitive to the polar temperature and thereby to obliquity. Climate models of $\mathrm{CO}_{2}$ evolution over obliquity changes which include exchange between atmospheric, polar caps, and regolith reservoirs suggest the possibility of large variations in atmospheric pressure (Ward et al. 1974, Toon et al. 1980, François et al. 1990, Fanale and Salvail 1994). Depending on the total inventory of available $\mathrm{CO}_{2}$, occasional or prolonged periods of warmer, more clement, climate may have resulted throughout geological history (Jakosky et al. 1995).

In the same manner, the transport and redistribution of water between ground ice, surface ice, and atmospheric reservoirs appear to be largely sensitive to orbital parameters. Surface and near-surface ice stability is most strongly controlled by obliquity through the variations of the surface thermal forcing and of the abundance of water vapor sublimed in summer (e.g. Mellon and Jakosky 1995; Jakosky et al. 1995). Simplified climate models suggest that during periods of high obliquity $\left(>40^{\circ}\right)$, large quantities of polar ice could be sublimed and transported away to the tropical regions where it becomes stable (Jakosky and Carr 1985, Jakosky et al. 1995). Such predictions were recently confirmed by full three-dimensional climate simulations (Haberle et al. 2000, Richardson and Wilson 2000, 2002, Mischna et al. 2003, Levrard et al. 2003). Conversely, at present obliquities and lower, water ice is expected to be stable only in the high-latitude areas.

Many geological features provide the possibility of recent orbital-driven climatic changes. The most impressive is the extensive layering observed in the polar deposits and thought to contain alternate layers of water ice and dust in different proportions. Correlation between stratigraphic sequences and insolation parameters suggests that polar caps may preserve climatic records spanning the last few millions of years (Laskar et al. 2002). Additional morphological evidence may be found in Mars Global Surveyor observations of suspected recent water (gullies, paleolakes, outflow channels) or ice-generated (contractioncrack polygons, paleo-glaciers) landforms (e.g. Baker 2001, Mustard et al. 2001, Costard et al. 2002, Head

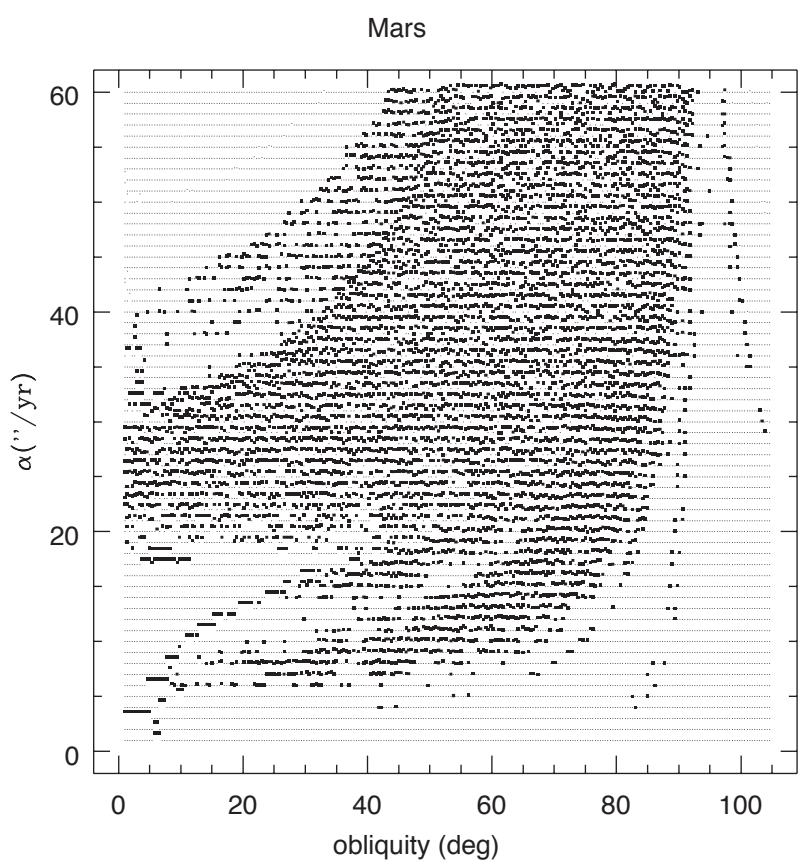

Figure 1: Chaotic zone for the obliquity of Mars. The x-axis is the initial obliquity (in degrees) and the y-axis is the precession constant $\alpha$, in $\operatorname{arcsec} / y r$. The regular solutions are represented by small dots, while large black dots denote the chaotic solutions (adapted from Laskar and Robutel 1993).

and Marchant 2003).

The presence of more ancient equatorial valley networks and putative oceanic shorelines, which support the occurrence of flowing water and different environmental conditions in early Mars (e.g. Baker et al. 1991), illustrates the extreme climate changes that Mars may have undergone throughout its geological history. In this context, longterm evolution and amplitudes of insolation parameters are a key element to understand the evolution of Martian surface processes.

The history of the computation of the astronomical solution for the forcing of Martian paleoclimates is very similar to what happened in the Earth's case (see Imbrie and Imbrie 1979), but it occurred over a shorter time. The first climate models took only into consideration the precession of the axis of the planet, that alters the seasonal contrast (Leighton and Murray 1966). Murray et al. (1973) then realized that the change of eccentricity of the planet resulting from secular planetary perturbations (from 0.004 to 0.141 in the solution of Brouwer and Van Woerkom (1950) that was then used), will modify significantly the insolation at the surface of the planet. The Earth spin axis obliquity undergoes variations of about $\pm 1.3^{\circ}$ around its mean value $\left(23.3^{\circ}\right.$ ) (Laskar et al. 1993 
and references therein) that are now recognized to have a large impact on the past climate of the Earth (see Imbrie and Imbrie 1979). Ward $(1973,1974)$ was the first to realize that the obliquity of Mars suffers much larger variations, due to the proximity of secular spin orbit resonances. Using the orbital solution of Brouwer and Van Woerkom (1950) he found that the obliquity $(\varepsilon)$ of Mars was oscillating between $\simeq 14.9^{\circ}$ and $\simeq 35.5^{\circ}$. This solution was later on improved, using the secular orbital solution of Bretagnon (1974), which led to slightly larger variations of the obliquity (Ward 1979). A significant change arose with the use of the secular solution of Laskar (1988). Using this orbital solution, Ward and Rudy (1991) found that the obliquity evolution of Mars was critically dependent on the initial precession rate, which was not known very precisely (see section 3.2). The reason for this behavior was explained when it was demonstrated that the evolution of Mars' obliquity is chaotic (Laskar and Robutel 1993, Touma and Wisdom 1993). Moreover, (Laskar and Robutel 1993) provided a global view for the dynamics of Mars' obliquity, describing the shape and extent of the associated chaotic zone (Figs. 1, 13). With this global portrait, and although the computations were only conducted over $45 \mathrm{Myr}$, they concluded that Mars' obliquity can wander from $0^{\circ}$ to more than $60^{\circ}$.

Since then, the most significant improvement for the computation of an astronomical solution for Martian paleoclimate studies is in the determination of the initial precession rate by the Pathfinder (Folkner et al. 1997) and Mars Global Surveyor space missions (Yoder et al. 2003), which now permit a reliable obliquity solution for Mars over a few million years.

After the pioneering work of Quinn et al. (1991), and Sussman and Wisdom (1992), and with the improvement of computer speed in the last decade, it becomes now possible numerically to integrate over hundreds of million of years (Myr) a dynamical model for the orbital evolution of the planetary orbits that is close to the ones used for short time ephemerides computations (Laskar 2001, Laskar et al. 2004, Varadi et al. 2003). In the first section, we will present the derivation of the orbital solution for Mars over 250 Myr using the new symplectic integrator developed in our group (Laskar and Robutel 2001). Particular care is taken to reduce the roundoff error, and the solution is compared to the most up-to-date numerical ephemeris DE406 (Standish 1998). The second part is devoted to the precession and obliquity equations and to the stability of the solutions with respect to the uncertainty of the parameters, and in particular of the initial precession rate. Because of the chaotic behavior of the obliquity, a precise solution cannot be derived over more than 10 to 20 Myr, but the equations are integrated over
$250 \mathrm{Myr}$ in order to establish statistics on the possible variations of the obliquity over this time span. In section 5 , this analysis is then continued over 5 Gyr. Over such an extended time, we do not use the direct numerical integration, but the secular equations of Laskar (1990), after some small adjustment of the parameters. This allows us to increase the computer speed by a factor of 2000 and to perform more extensive statistics, on both the orbital and obliquity solutions. We are then able to derive simple analytical expressions that fit extremely well with the density distribution of the eccentricity and obliquity over 5 Gyr (section 5.4). In this sense, thanks to the chaotic behavior of the solutions, we can make very precise predictions on the obliquity and eccentricity evolution beyond $500 \mathrm{Myr}$, but these predictions are not for the exact values of these quantities, but for their probabilistic density functions.

\section{Evolution over $250 \mathrm{Myr}$}

In this first part, we will use a direct numerical integration of the planetary orbital motion in order to investigate the behavior of Mars' obliquity over 250 Myr. As the orbital motion is chaotic, even with a precise dynamical model, the computer roundoff numerical error alone will prevent obtaining a precise orbital solution for Mars over more than 60 Myr (see Fig.4.a). Moreover, the obliquity of Mars itself is chaotic, even more chaotic than its orbital motion (Laskar and Robutel 1993, Touma and Wisdom 1993). This will prevent even more drastically obtaining a precise solution for the obliquity over more than 10 to 20 Myr, with the present knowledge of the initial parameters (section 3.2.1).

Our goal in this section will thus be to obtain a solution for the insolation parameters of Mars as precise as possible over 10 to 20 Myr for use in Mars paleoclimate studies. Then, with the same model, to explore the behavior of the solutions over $250 \mathrm{Myr}$ and to derive a statistical vision of this chaotic system. Although no precise prediction is possible over this time interval, we will be able to derive a precise estimate of the density probability function for the evolution of the eccentricity of Mars and its obliquity. It is in fact paradoxical (see for example Lasota and Mackey 1994) that it is actually the chaotic behavior of the system that will allow us to make a precise prediction of the evolution of the density function of Mars' orbital and rotational parameters.

\subsection{Orbital Motion}

The orbital model comprises all 9 main planets of the Solar System, including Pluto. The post-newtonian general 
relativity corrections of order $1 / c^{2}$ due to the Sun are included following Saha and Tremaine (1994).

The Moon is treated as a separate object. In order to obtain a realistic evolution of the Earth-Moon system, we also take into account the most important coefficient $\left(J_{2}\right)$ in the gravitational potential of the Earth and of the Moon, and the tidal dissipation in the Earth-Moon system. We also integrate at the same time the precession and obliquity equations for the Earth and the evolution of its rotation period in a comprehensive and coherent way, following the lines of Néron de Surgy and Laskar (1997) and of Correia et al. (2003). More details on the integration model can be found in (Laskar et al. 2004).

\subsection{Numerical integrator}

In order to minimize the accumulation of roundoff error, the numerical integration was performed with the new symplectic integrator scheme $S A B A C_{4}$ of Laskar and Robutel (2001), with a correction step for the integration of the Moon. This integrator is particularly adapted to perturbed systems where the Hamiltonian governing the equations of motion can be written on the form $H=A+\epsilon B$, as the sum of an integrable part $A$ (the Keplerian equations of the planets orbiting the Sun), and a small perturbation potential $\epsilon B$ (here the small parameter $\epsilon$ is of the order of the planetary masses). Using this integrator with step size $\tau$ is then equivalent to integrating exactly a nearby Hamiltonian $\tilde{H}$, where the method's error $H-\tilde{H}$ is of the order of $O\left(\tau^{8} \epsilon\right)+O\left(\tau^{2} \epsilon^{2}\right)$, and even $O\left(\tau^{8} \epsilon\right)+O\left(\tau^{4} \epsilon^{2}\right)$ when the correction step is added, while the same quantity is of the order $O\left(\tau^{2} \epsilon\right)$ in the widely used symplectic integrator of Wisdom and Holman (1991), and $O\left(\tau^{n} \epsilon\right)+O\left(\tau^{2} \epsilon^{2}\right)$ with the correctors of Wisdom et al. (1996).

The step size used in most of the integration is $\tau=$ $5 \times 10^{-3} \mathrm{yr}=1.82625$ days. The initial conditions of the integration were least-square adjusted to the JPL ephemeris DE406 (Standish 1998), in order to compensate for small differences in the model. In particular, we do not take into account the effect of the minor planets, and the modeling of the interactions in the Earth-Moon system is more complete in DE406 (see Williams et al. 2001).

In Figure 2.a is plotted the evolution of the total energy of the system from $-250 \mathrm{Myr}$ to $+250 \mathrm{Myr}$, after the removal of the secular trend that corresponds to the dissipation in the Earth-Moon system. The residuals are smaller than $2.5 \times 10^{-10}$ after $250 \mathrm{Myr}$, and behave as a random walk with a standard deviation per step $\sigma_{1} \approx 2.7 \epsilon_{M}$, where $\epsilon_{M} \approx 2.22 \times 10^{-16}$ is the machine Epsilon in double precision (Laskar et al. 2003). The normal component of

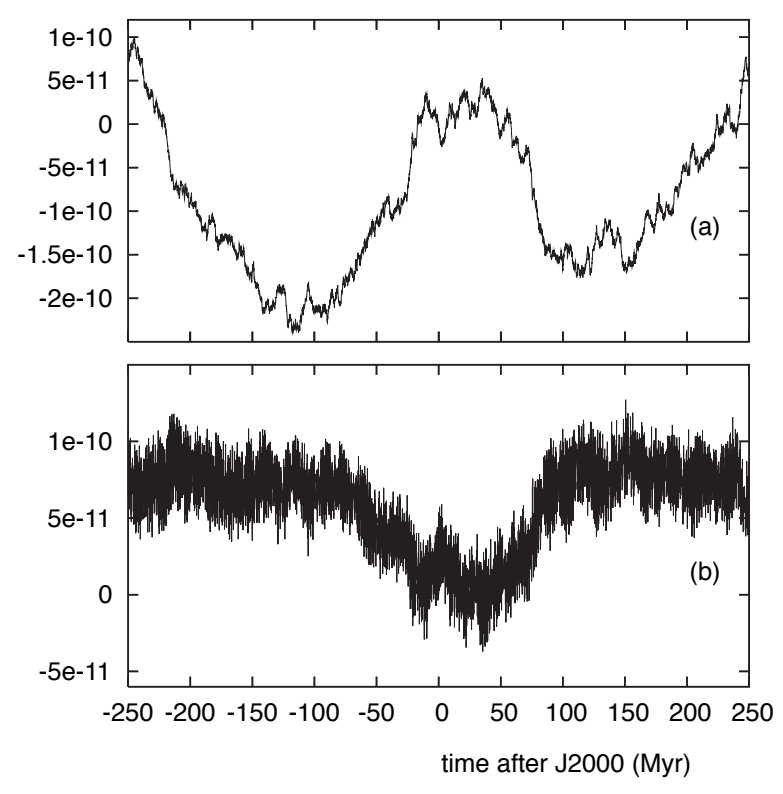

Figure 2: Integrals conservation. (a) relative variation of the total energy of the system versus time (in Myr) from -250 Myr to + 250 Myr (with origin at J2000) after correction of the secular trend due to the tidal dissipation in the Earth-Moon system. (b) relative variation of the normal component of the total angular momentum.

the angular momentum is conserved over the same time with a relative error of less than $1.3 \times 10^{-10}$ (Fig.2.b).

With these settings, the CPU time on a Compaq alpha workstation $(833 \mathrm{Mhz}$ ) is about $24 \mathrm{~h}$ for $5 \mathrm{Myr}$, and a 250 Myr run will last nearly two months.

\subsection{Comparison with DE406}

Using a direct numerical integrator, our goal is to provide a long term solution for the orbital and precessional elements of Mars with a precision that is comparable with the usual accuracy of a short time ephemeris.

We have thus compared our solution with the most advanced present numerical integration, DE406, that was itself adjusted to the observations (Standish 1998). In the present paper, we will only discuss the orbital solution of Mars (Fig.3).

Over the full range of DE406, that is from $-5000 \mathrm{yr}$ to + $1000 \mathrm{yr}$ from the present date, the maximum difference in Mars' longitude is less than 0.28 arcsec. These differences probably account in large part for the perturbations by the minor planets that are not taken into account in our computations. Over the whole interval, the difference in eccentricity is less than $5 \times 10^{-8}$, and less than 0.08 arcsec in inclination. 


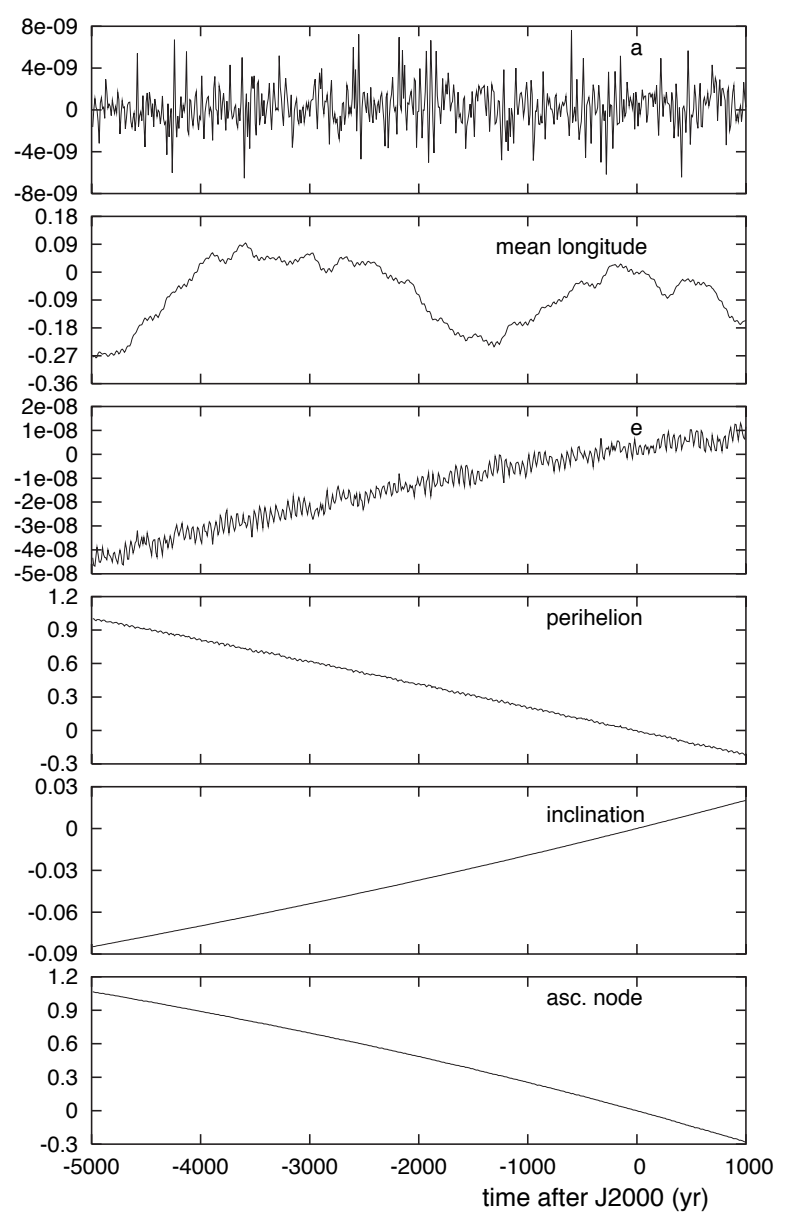

Figure 3: Differences La2003-DE406 over the full range of DE406 $(-5000 \mathrm{yr}$ to $+1000 \mathrm{yr}$ from J2000) for the orbital solution of Mars for all elliptical elements $(a, \lambda, e, \varpi, i, \Omega)$ respectively. The units for semi-major axis $(a)$ are $\mathrm{AU}$, and arcsec for mean longitude $(\lambda)$, longitude of perihelion $(\varpi)$, inclination $(i)$, and longitude of the ascending node $(\Omega)$ from the ecliptic and equinox J2000.

\subsection{Variations on the orbital model}

We will not present here all the wanderings that we had in the past years while searching for a precise model, which led us to obtain several intermediate solutions. A general discussion of the sensitivity of the orbital solution to the model, resulting from the chaotic behavior of the solution can be found in (Laskar 1999a). Although this analysis was done with the secular equations, the conclusions would probably not be much changed using the complete equations.

The most recent determination of the Solar oblateness $\left(J_{2}\right)$, obtained with the SOHO and GONG helioseismic data give $J_{2}=(2.18 \pm 0.06) \times 10^{-7}$ (Pijpers 1998), with a very similar value adopted in $\operatorname{DE} 406\left(J_{2}=2 \times 10^{-7}\right.$,
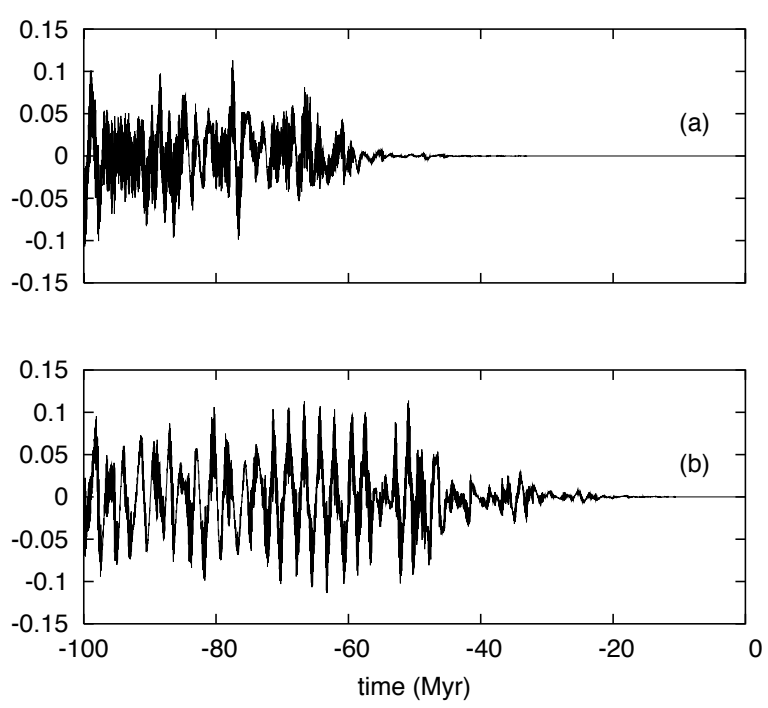

Figure 4: Stability of the solution for Mars' eccentricity. (a) Difference of the nominal solution La2003 with stepsize $\tau=5 . \times 10^{-3}$ years, and La2003*, obtained with $\tau^{*}=4.8828125 \times 10^{-3}$ years. (b) Difference of the nominal solution with the solution obtained while setting $J_{2}=0$ for the Sun (instead of $2 \times 10^{-7}$ in the nominal solution).

Standish 1998), while in DE200, it was not taken into account (Newhall et al. 1983). Even with this small value, the absence of the Solar oblateness in the dynamical model was identified in (Laskar 1999a) as one of the main source of uncertainty in the long term solution for the Solar System. We will thus consider that comparing the nominal solution La2003 (with $J_{2}=2 \times 10^{-7}$ ) with an alternate solution (La20030) with $J_{2}=0$ is representative of the uncertainty of the dynamical model for our long term integrations.

The results La2003 - La2003 0 for Mars' eccentricity are displayed in Figure 4.b over $100 \mathrm{Myr}$. The effect of the $J_{2}$ becomes noticeable after about 30 Myr (26 Myr were predicted with an analytical estimate in (Laskar 1999a)), and the solution remains very similar over $40 \mathrm{Myr}$, and totally out of phase after $45 \mathrm{Myr}$. We will thus consider here that $40 \mathrm{Myr}$ is about the time of validity of our present orbital solution for Mars.

In Fig. 4.a, we have also tested the numerical stability of our numerical integration. This is done by comparison of the nominal solution La2003 (with stepsize $\tau=5 \times 10^{-3}$ years) with an alternate solution, La2003*, with the same dynamical model, and a very close stepsize $\tau^{*}=4.8828125 \times 10^{-3}$ years. This special value was chosen in order that our output time span $h=1000$ years corresponds to an integer number (204800) of steps, in order to avoid any interpolation problems in the check of 
the numerical accuracy. Fig. 4.a is thus a test of the time of validity for the obtention of a precise numerical solution with a given dynamical model, which is thus limited here to about $60 \mathrm{Myr}$.

This limitation of $60 \mathrm{Myr}$ is at present a limitation for the time of validity of an orbital solution, independently of the precision of the dynamical model. In order to go beyond this limit, the only way will be to increase the numerical accuracy of our computations, by improving the numerical algorithm, or with an extended precision for the number representation in the computer. It should be noted that with our present algorithms, we are much more limited by the precision of the model (Fig. 4.b) than by the numerical accuracy (Fig. 4.a).

At this point, we need to stress that after showing that the solution is probably not precise over more than 40 Myr, and certainly not after $60 \mathrm{Myr}$, we are going in the remaining part of this paper to compute solutions over $250 \mathrm{Myr}$ with the same algorithm (Fig. 5), and even 5 Gyr with the secular equations. This is indeed justified, as in this case we will not pretend to provide the actual solution for the evolution of the Solar System, but just investigate its possible behavior.

Finally, as it will be shown in the next sections, because of the strong chaos of the obliquity evolution of Mars, and of the uncertainties on its initial conditions, the obliquity solution will only be valid over 10 to $20 \mathrm{Myr}$, that is over much less time than the orbital computation. Over such a short time, the orbital present solution is certainly computed with a very good accuracy (Fig.4.b).

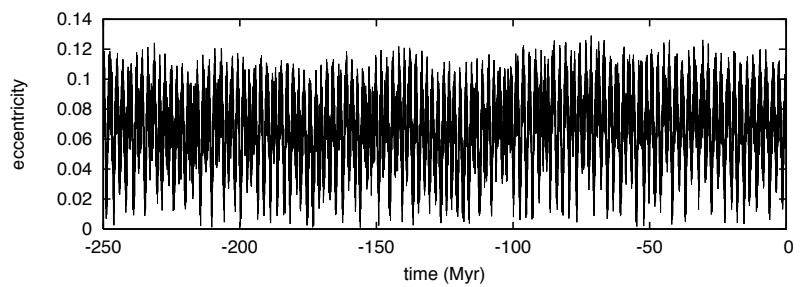

Figure 5: The nominal solution La2003 for Mars' eccentricity over 250 Myr.

\section{Precession equations}

The equations for the evolution of precession and obliquity of Mars are rather simple, as contrary to the Earth's case, the effect of the satellites can be neglected. Indeed, it was shown by Goldreich (1965) that when a satellite is close to its planet, its orbit precesses about the planet's equatorial plane, as it is the case for Phobos and Deimos.
In this case, the averaged torque exerted by the satellite is zero (see Laskar 2004).

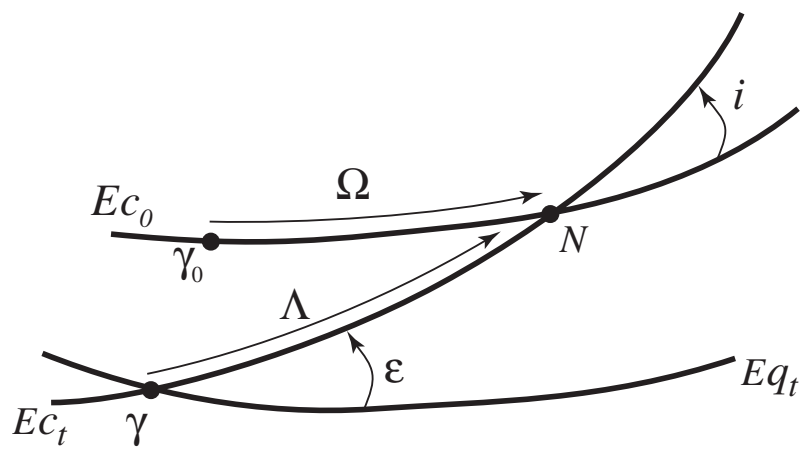

Figure 6: Fundamental planes for the definition of precession and obliquity. $E q_{t}$ and $E c_{t}$ are the mean equator and ecliptic of Mars at date $t . E c_{0}$ is the fixed ecliptic of the Earth at Julian date J2000, with equinox $\gamma_{0}$. The general precession in longitude $\psi$ is defined by $\psi=\Lambda-\Omega$. $\Omega$ is the longitude of the node, and $i$ the inclination. The angle $\varepsilon$ between $E q_{t}$ and $E c_{t}$ is the obliquity.

We suppose here that Mars is an homogeneous rigid body with moments of inertia $A<B<C$ and we assume that its spin axis is also the principal axis of greatest inertia. The precession $\psi$ and obliquity $\varepsilon$ (Fig. 6) equations for a rigid planet in the presence of planetary perturbations are given by (Kinoshita 1977, Laskar et al. 1993 , Néron de Surgy and Laskar 1997):

$$
\left\{\begin{array}{l}
\frac{d \varepsilon}{d t}=-\mathcal{B}(t) \sin \psi+\mathcal{A}(t) \cos \psi \\
\frac{d \psi}{d t}=\alpha \cos \varepsilon-\cot \varepsilon(\mathcal{A}(t) \sin \psi+\mathcal{B}(t) \cos \psi)-2 \mathcal{C}(t)
\end{array}\right.
$$

with

$$
\left\{\begin{array}{l}
\mathcal{A}(t)=\frac{2}{\sqrt{1-p^{2}-q^{2}}}[\dot{q}+p(q \dot{p}-p \dot{q})] \\
\mathcal{B}(t)=\frac{2}{\sqrt{1-p^{2}-q^{2}}}[\dot{p}-q(q \dot{p}-p \dot{q})] \\
\mathcal{C}(t)=q \dot{p}-p \dot{q}
\end{array}\right.
$$

where $q=\sin (i / 2) \cos \Omega$ and $p=\sin (i / 2) \sin \Omega$, and where $\alpha$ is the "precession constant":

$$
\alpha=\frac{3 G}{2 \omega} \frac{m_{\odot}}{\left(a \sqrt{1-e^{2}}\right)^{3}} E_{d}
$$

where $m_{\odot}$ is the solar mass, $G$ the gravitational constant, $\omega$ the rotation rate of the planet, and $E_{d}=(2 C-A-$ $B) / 2 C$ its dynamical ellipticity. For a fast rotating planet like Mars, $E_{d}$ can be considered as proportional to $\omega^{2}$; this corresponds to hydrostatic equilibrium (see for example 
Lambeck, 1980). In this approximation, $\alpha$ is thus proportional to $\omega$. The quantities $\mathcal{A}, \mathcal{B}$ and $\mathcal{C}$ describe the driving terms due to the secular evolution of the orbital plane of the planet and are given by the integration of the planetary motions.

\subsection{Dissipative effects}
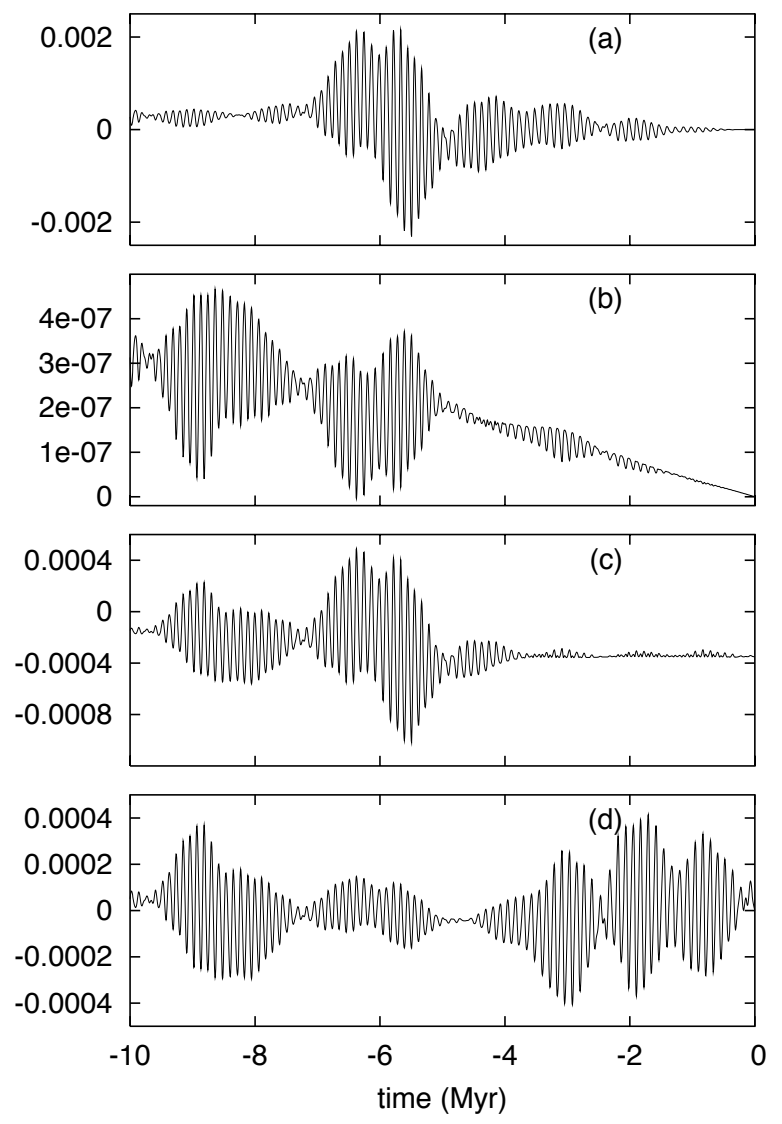

Figure 7: Influence of parameter variation. The change (in degrees) observed over $10 \mathrm{Ma}$ for the various parameters. (a) effect of tidal dissipation; (b) core mantle friction; (c) $10 \times$ the uncertainty in the initial obliquity; (d) $10 \times$ the uncertainty in the initial precession angle.

The possible dissipative effects influencing the evolution of Mars' spin axis are : tidal dissipation due to the Sun, core-mantle friction, and climate friction.

\subsubsection{Tidal dissipation}

We have considered the tidal contribution from the Sun, following the equations given in (Néron de Surgy and Laskar 1997, Correia et al. 2003). Tidal contributions from the satellites Phobos and Deimos can be neglected compared with solar tides (Lambeck 1979, Mignard 1981). As shown in Fig. 7a, the effect of solar tidal dissipation is also very small, as it amounts to less than 0.002 degrees after $10 \mathrm{Ma}$. As a consequence, the rotation rate of the planet did not change very much since the beginning of the Solar System. The choice of the tidal dissipation model is then not very relevant and we decided to use for Mars a constant-Q model (see Correia et al. 2003, for the explicit equations ). For this model, the dissipation is independent of the rotation rate, and its amplitude is proportional to $k_{2} \sin (2 \omega \Delta t) \simeq k_{2} / Q$, where $\Delta t$ is the time delay between the tidal perturbation from the Sun and the consequent deformation of the planet. We used for the nominal solution La2003 a second Love number $k_{2}=0.14$ and a dissipative factor $Q=50$, while for the more recent La2004 solution (see section 3.2.2) we used $k_{2}=0.149$ and $Q=92$ from (Yoder et al. 2003). In both cases, the tidal contribution remains very small.

\subsubsection{Core-mantle friction}

Little is known about the interior of Mars, since there are few geophysical constraints on its internal structure. However, the presence of a core can be inferred by the polar moment of inertia (Cook 1977, Zhang 1994). The existence of remanant crustal magnetism (Acuña et al. 1999) and the best determination of the polar moment of inertia so far (Folkner et al. 1997) imply that Mars' core has substantial iron and that at least the outer part is liquid (Yoder et al. 2003). Because of their different densities, the core and the mantle do not have the same dynamical ellipticity. Since the precession torques exerted by the Sun on Mars' core and mantle are proportional to this quantity, the two parts tend to precess differently around an axis perpendicular to the orbital plane (this results from Poincaré's study (1910) on the motion of an inviscid fluid contained in a rotating ellipsoidal shell). This tendency is more or less counteracted essentially by two different interactions produced at the interface: the torque of nonradial inertial pressure forces of the mantle over the core provoked by the non-spherical shape of their interface; and the torque of the viscous friction between the core and the mantle (e.g. Correia et al. 2003). The effect of core viscosity in the Earth's case was treated by Stewartson and Roberts (1963) and Roberts and Stewartson (1965) for low values of viscosity, by linearizing the equations for the viscous boundary layer. Busse (1968) further studied the effect of the non-linear advective term in the equations. The contribution of core-mantle friction to the secular variation of the obliquity is given by (Rochester 
1976, Pais et al 1999):

$$
\dot{\varepsilon} \simeq-\kappa \frac{\alpha^{2} \cos ^{3} \varepsilon \sin \varepsilon}{\gamma_{e l} C E_{c}^{2} \omega^{2}},
$$

with (Roberts and Stewartson 1965, Busse 1968)

$$
\kappa \simeq 2.62 \frac{C_{c} \sqrt{\omega \nu}}{R_{c}},
$$

where $C_{c}, E_{c}$ and $R_{c}$ are respectively the core's polar moment of inertia, dynamical ellipticity and radius; $\nu$ is the kinematic viscosity and $\gamma_{e l} \simeq 0.75$ a correcting factor accounting for the elastic deformation of the mantle. The kinematic viscosity $(\nu)$ is poorly known. Even in the case of the Earth, its uncertainty covers about 13 orders of magnitude (Lumb and Aldridge 1991). It can be as small as $\nu=10^{-7} \mathrm{~m}^{2} \mathrm{~s}^{-1}$ for the Maxwellian relaxation time and experimental values for liquid metals or as big as $\nu=10^{5} \mathrm{~m}^{2} \mathrm{~s}^{-1}$ for the damping of the Chandler wobble or attenuation of shear waves. The best estimate so far of the actual value of this parameter is $\nu \simeq 10^{-6} \mathrm{~m}^{2} \mathrm{~s}^{-1}$ (Gans 1972, Poirier 1988). The secular obliquity variations given by expression (4) are of the same order as the tidal variations for the highest values of the viscosity and can be neglected for the best estimations. Indeed, with $Q=100$ we compute for tidal evolution:

$$
\dot{\varepsilon}_{\text {tides }} \sim 10^{-7} \operatorname{arcsec} / \mathrm{yr} .
$$

Using $R_{c}=1.68 \times 10^{6} \mathrm{~m}$ (Yoder et al. 2003), $C_{c}=0.06 C$ (we compute $C_{c}=8 \pi \rho_{\mathrm{Fe}} R_{c}^{5} / 15$, with $\rho_{\mathrm{Fe}} \simeq 7 \mathrm{~g} / \mathrm{cm}^{3}$ ) and $E_{c}=0.5 E_{d}$ (we compute $E_{c} / E_{d}=C R_{c}^{5} / C_{c} R^{5}$ ), we have for secular obliquity variations resulting from core-mantle friction:

$$
\dot{\varepsilon}_{\mathrm{cmf}} \sim 10^{-7} \sqrt{\nu_{\left[\mathrm{m}^{2} \mathrm{~s}^{-1}\right]}} \operatorname{arcsec} / \mathrm{yr} .
$$

For weak viscosity like the best estimations for the Earth $\left(\nu \sim 10^{-6} \mathrm{~m}^{2} \mathrm{~s}^{-1}\right)$, we have $\dot{\varepsilon}_{\mathrm{cmf}} \sim 10^{-3} \dot{\varepsilon}_{\text {tides }}$, that is, the core-mantle friction effect can be neglected with respect to solar tides (Fig. 7.b). Since this parameter is very uncertain, we can expect that electromagnetic coupling or turbulence to increase friction at the interface between the core and the mantle. We can then talk about an effective viscosity some orders of magnitude higher, but we see that even for $\nu \sim 1 \mathrm{~m}^{2} \mathrm{~s}^{-1}$, we still have $\dot{\varepsilon}_{\mathrm{cmf}} \sim \dot{\varepsilon}_{\text {tides }}$. The core-mantle friction effect is then of the same order of tidal effects or even weaker.

\subsubsection{Climate friction}

Climate friction is a positive feedback process between obliquity variations and the resulting redistribution of volatiles at the planetary surface that affect its dynamical ellipticity. Although a significant fraction of the variations of surface loading is compensated by the visco-elastic adjustment of the internal planetary mass, delayed responses in climatic and viscous relaxation processes may lead to a secular term in the obliquity evolution. Conversely to previous dissipative effects, climate friction not only depends on the instantaneous value of the obliquity but also on its dynamical evolution.

The impact of $\mathrm{CO}_{2}$ and water caps has been respectively studied by Rubincam (1990, 1993) and Bills (1999) using linear approximations for the obliquity dynamics and global mass redistribution. Because both the volatile response to obliquity forcing and Martian internal parameters (density, elasticity, rigidity, viscosity) are still poorly constrained, long-term estimation of the climate friction impact is very uncertain. Similar analyses suggest that obliquity-oblateness feedback has probably not changed the Earth's obliquity by more than $0.01^{\circ} / \mathrm{Myr}$ during the severe recent Pliocene-Pleistocene glaciations $(\sim 0-3 \mathrm{Ma})$ (Levrard and Laskar, 2003). Since the Martian caps are about one order of magnitude less massive than the water/ice mass involved in typical terrestrial ice age, we expect that its impact is negligible over the last $20 \mathrm{Myr}$, compared to other sources of uncertainty, and it was not taken into account in our long-term obliquity solutions.

Other geophysical processes, such as volcanic events and uplifts, construction of the large Tharsis province or Hellas impact basins, or formation of mass anomalies associated with mantle convection could have produced changes of early Mars' obliquity (Ward et al. 1979), but they do not affect the recent 20 Myr of Martian history.

\subsection{Initial spin axis orientation}

As the motion of Mars' spin axis is chaotic (Laskar and Robutel 1993, Touma and Wisdom 1993), its evolution critically depends on its precise initial conditions. Significant improvements were made on this determination with the results of the Pathfinder mission (Folkner et al. 1997). Folkner et al. (1997) provided some estimates on the determinations of the initial conditions for the spin of Mars (Table 1). According to their paper, this uncertainty represents five times the standard error $(\sigma)$ obtained during their fitting process to the data. This is more than what is usually done $(3 \sigma)$, but according to the authors, this allows for 'the failure to account for various systematic effects'. We will be thus even more cautious on these data, and investigate its possible variations up to $10 \sigma$, that is $2 \delta p$, around $p_{0}$ where

$$
p_{0}=-7.576 \mathrm{arcsec} / \text { year and } \delta p=0.035 \mathrm{arcsec} / \text { year }
$$


are the initial value of the precession rate and its uncertainty given in Table 1.

\begin{tabular}{cc}
\hline Parameter & Value \\
\hline Obliquity $\varepsilon$ (degrees) & $25.189417(35)$ \\
Node $\psi$ (degrees) & $35.43777(14)$ \\
Precession rate $d \psi / d t$ (mas/year) & $-7576(35)$ \\
Rotation rate $\omega$ (degrees/day) & $350.89198521(8)$ \\
\hline
\end{tabular}

Table 1: Initial conditions from (Folkner et al. 1997). The uncertainty is given in parentheses in the units of the last displayed digit.
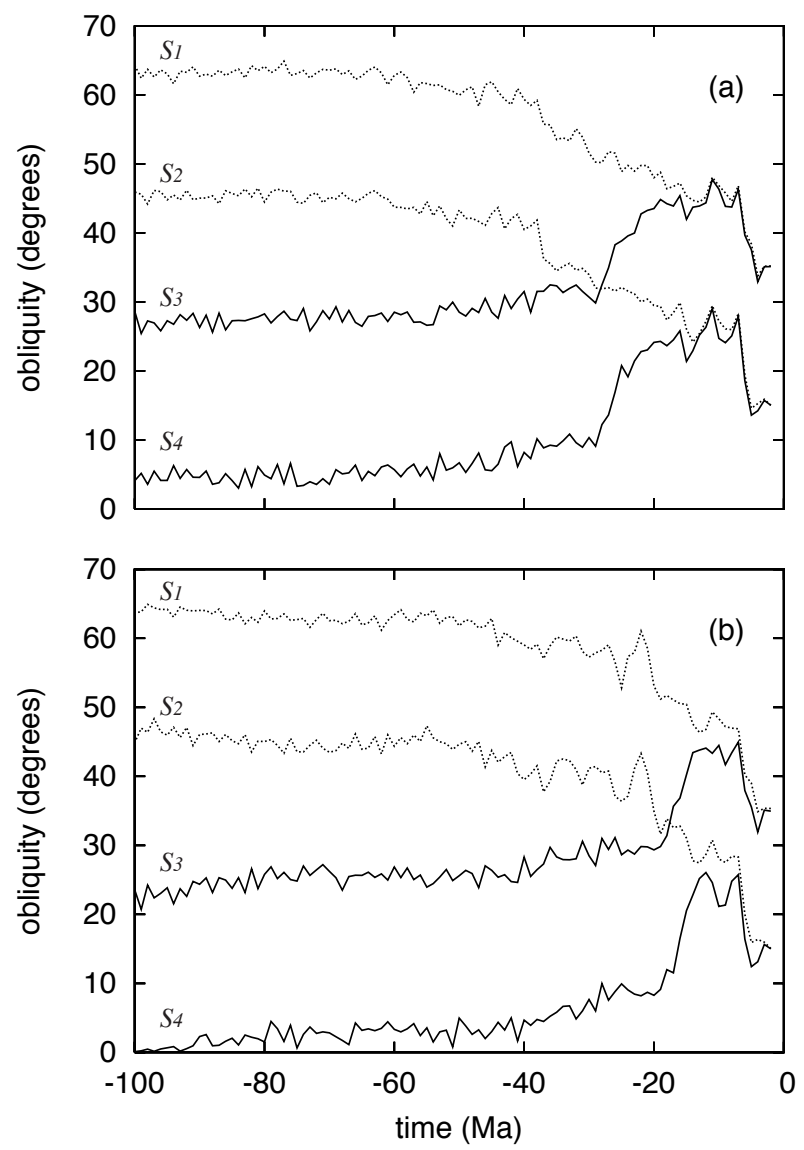

Figure 8: Variations in the obliquity solution due to the uncertainty in the precession frequency. In (a), 1001 solutions of the obliquity are computed, with initial precession values ranging from $p_{0}-\delta p / 2$ to $p_{0}+\delta p / 2$ with an even stepsize. In (b) the same analysis is made with 401 solutions with initial precession values ranging from $p_{0}-2 \delta p$ to $p_{0}+2 \delta p$. The maximum $M_{k}$ and minimum $m_{k}$ envelopes over $1 \mathrm{Myr}$ are computed for each solution $k$. The plotted curves are then : $S_{1}=\max \left(M_{k}\right), S_{2}=\max \left(m_{k}\right), S_{3}=\min \left(M_{k}\right), S_{4}=$ $\min \left(m_{k}\right)$.

First of all, we will show that the uncertainty on the initial position of the pole axis is sufficiently small and does not induce significant changes in the solution. We have thus run some numerical integrations with a deviation of 10 times this uncertainty in both the initial obliquity (Fig. 7.c) and precession angle (Fig. 7.d). In both cases, the maximum difference with respect to the nominal solution is less than $0.001^{\circ}$ after $10 \mathrm{Myr}$, and thus negligible, compared to other uncertainties.

\subsubsection{Precession constant}

In fact, the main source of uncertainty in the long term evolution of the obliquity of Mars is the uncertainty in the initial precession rate of Mars. Nevertheless, even when being pessimistic with respect to the accuracy of the published determination (Table 1), the situation after the Pathfinder mission is much improved, and it is now possible to derive results over $10 \mathrm{Myr}$ with confidence. In order to test the stability of the obliquity solution with respect to the uncertainty $\delta p$ of the initial precession frequency $p$, we have integrated 401 different solutions over $100 \mathrm{Myr}$, using always as an input the same orbital solution, and with an initial precession frequency $p_{k}=p_{0}+k \times \delta p / 100$, for $k=-200, \ldots, 200$. The different solutions are very similar in the vicinity of the origin, but after a few Myrs, they diverge significantly, as a result of the chaotic behavior of the spin precession, resulting from the secular perturbations of the other planets (Laskar and Robutel 1993).

In Figure 8b, we have summarized the obliquity evolution of all these solutions. For each solution $\varepsilon_{k}(t),(k=$ $-200, \ldots, 200)$, we have computed the maximum $M_{k}(t)$ and minimal value $m_{k}(t)$ reached by the obliquity over 1 Myr. We have then taken the upper and lower envelopes of these curves. The 4 curves are thus (in decreasing value at $100 \mathrm{Myr}) S_{1}=\operatorname{Max}\left(M_{k}\right), S_{2}=\operatorname{Max}\left(m_{k}\right), S_{3}=$ $\operatorname{Min}\left(M_{k}\right), S_{4}=\operatorname{Min}\left(m_{k}\right)$. They represent the extreme variations of the obliquity over $100 \mathrm{Myr}$. As was first shown in (Laskar and Robutel 1993), the variation of the obliquity ranges from $0^{\circ}$ to more than $60^{\circ}$.

The differences of the curves $S_{1}, S_{3}$ and $S_{2}, S_{4}$ represent the variability of the solutions. It is thus clear that if the uncertainty on the precession frequency $p$ is $2 \delta p$, the solution for the obliquity cannot be precise over more than $10 \mathrm{Myr}$.

We have repeated the same experience with a more optimistic view, with an initial precession frequency $p_{k}=$ $p_{0}+k \times \delta p / 1000$, for $k=-500, \ldots, 500$, that is on the interval $p_{0}-\delta p / 2, p_{0}+\delta p / 2$. In this case, the variation of the obliquity also ranges from $0^{\circ}$ to more than $60^{\circ}$, but the solution appears to be valid over nearly $20 \mathrm{Myr}$ (8.a).

Depending on the reliability of the uncertainty on $p_{0}$ given in Table 1, we can thus provide a reliable solution 
for the obliquity of Mars over 10 to 20 Myr. The nominal solution (denoted La2003) can be retrieved from the Web site http://www.imcce.fr/Equipes/ASD/mars.html from -20 Myr to +10 Myr (Fig.9) together with the subroutine for the computation of the various insolation quantities following Laskar et al. (1993). This is the solution that was used for the paleoclimate analysis of Mars polar cap layers in (Laskar et al. 2002). A very important constraint in this analysis was the observation that despite the uncertainty of the precession constant, and even using $2 \delta p$ for this uncertainty, all the solutions presented a large increase of the obliquity at $5 \mathrm{Myr}$ (Fig. 8b,10), already observed in some solutions of Ward and Rudy (1991) and Touma and Wisdom (1993), whose effect should have been noticeable on the ice record.

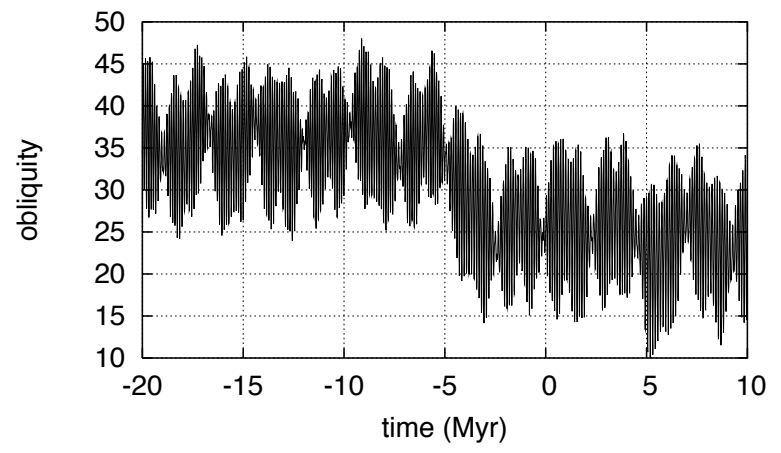

Figure 9: Obliquity (in degrees) of the nominal solution La2003 from -20 Myr to +10 Myr.

\subsubsection{The solution La2004}

The previous discussion demonstrates the importance of a better determination of Mars' initial precession rate, as this will be the only way to extend further our knowledge of the past insolation on the planet. We will thus be attentive to the next space missions to Mars with geodesic studies. The Netlander mission, initially projected in 2005, is a very good candidate for this goal (Barriot et al. 2001). One should consider that the determination with only the Viking mission gave $p=-7.83 \pm 0.3$ arcsec/year (probably $3 \sigma$ ) (Yoder and Standish 1997), while the addition of the Pathfinder mission gave $p=-7.576 \pm 0.035$ arcsec/year ( $5 \sigma)$. More recently, using the Mars Global Surveyor data, Yoder et al. (2003) gave a new determination of the precession constant as $p=-7.597 \pm 0.025$ arcsec/year. The uncertainty is still $5 \sigma$, and is slightly improved with respect to the previous determination. We have thus updated our solution using these new data. Moreover Yoder et al. (2003) give also an improved determination of the

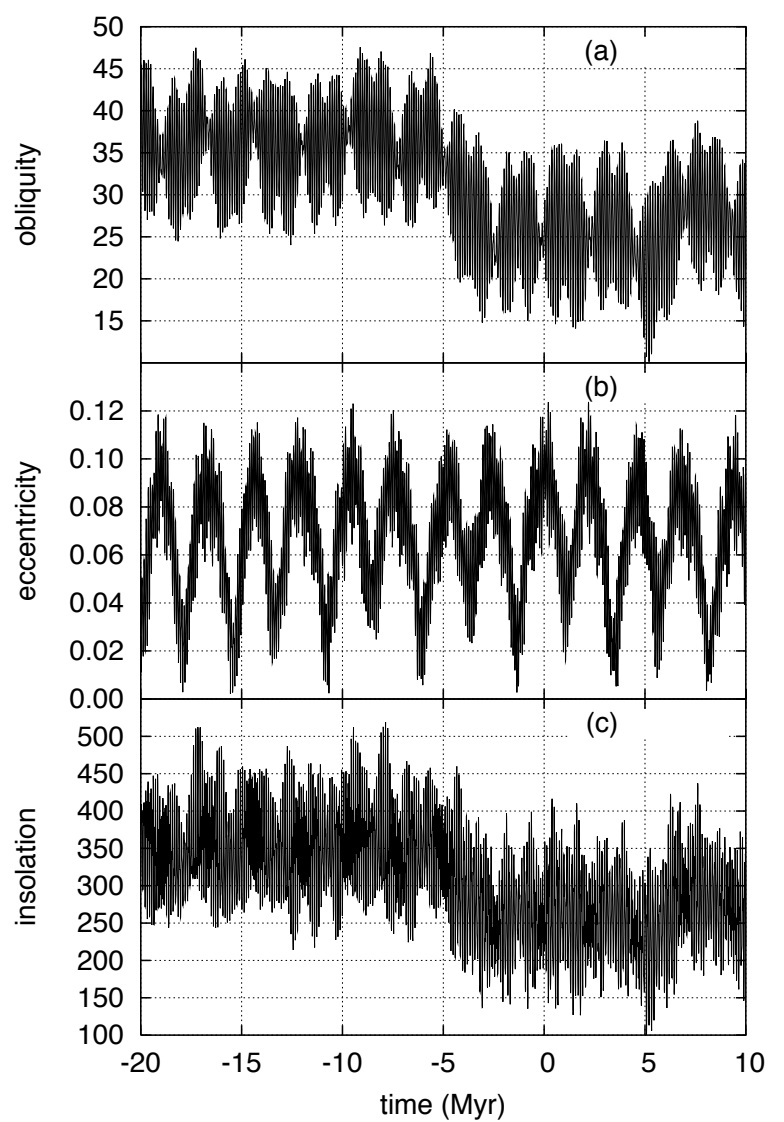

Figure 10: Obliquity (in degrees) (a), eccentricity (b), and insolation (c) (in watt $/ \mathrm{m}^{2}$ ) at the north pole surface at the summer solstice $\left(L_{S}=90^{\circ}\right)$ for the solution La2004 from $-20 \mathrm{Myr}$ to $+10 \mathrm{Myr}$.

tidal coefficients $k_{2}=0.149 \pm 0.017$ and $Q=92 \pm 11$ that are different from the values of our nominal solution $\left(k_{2}=0.14\right.$ and $\left.Q=50\right)$. As the tidal dissipation is very small (Fig. 7), this has no noticeable effect on the solution, but we have updated these data to their new values as well. The resulting solution will thus be called La2004, and will be provided as well on our Website http://www.imcce.fr/Equipes/ASD/mars.html.

The difference of the two solutions over $20 \mathrm{Myr}$ is small (compare Fig. 9 and Fig. 10a), and we have not done again the previous stability analysis with the updated values of La2004. The solution La2004 for the obliquity, eccentricity, and insolation in summer at the North pole is given in Fig. 10. 

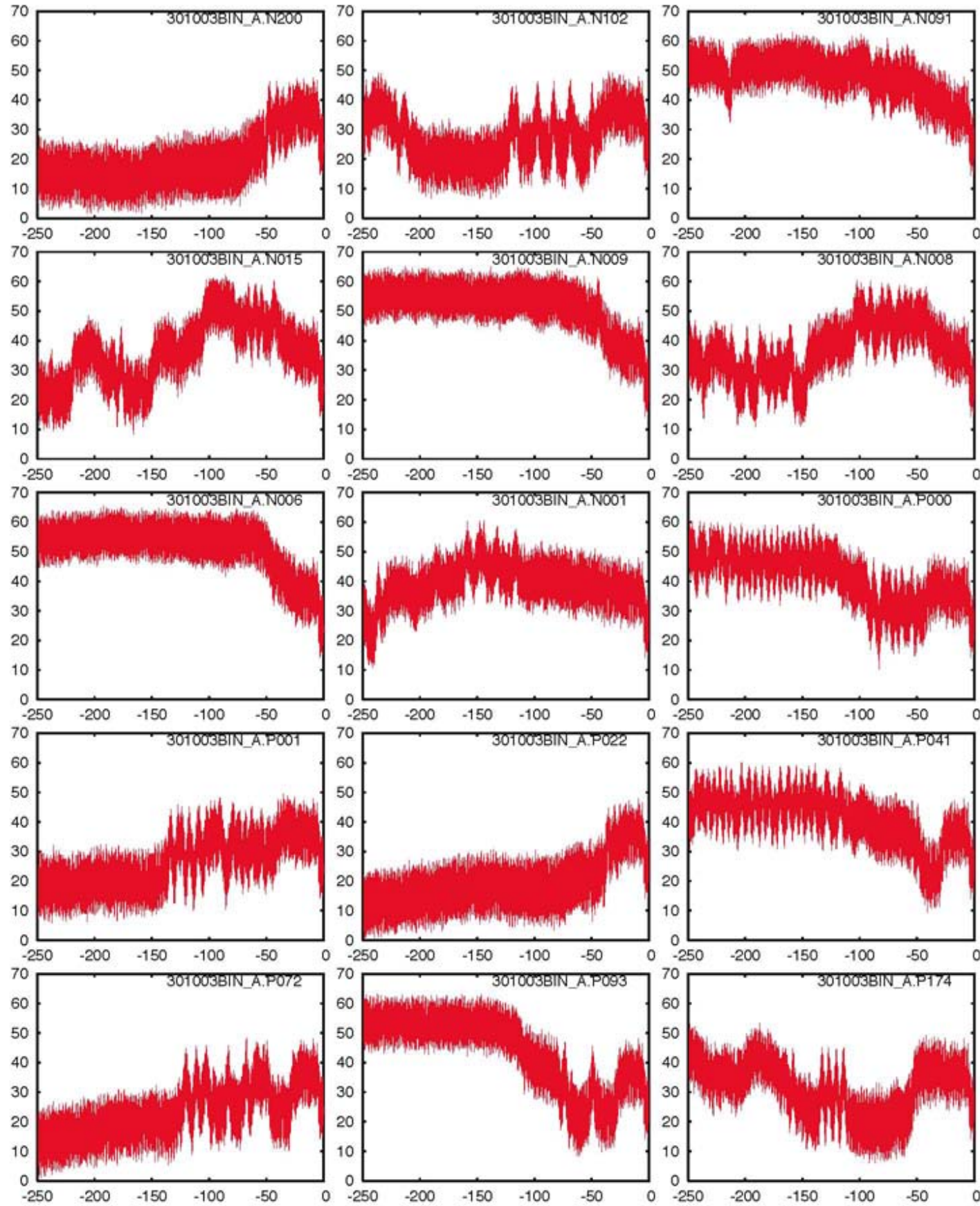

Figure 11: Examples of possible evolution of Mars' obliquity over the past $250 \mathrm{Myr}$. 


\section{Variations over $250 \mathrm{Ma}$}

We have plotted in Fig. 11 several examples of the solutions obtained by changing the initial precession rate $p$ by a small amount. In this figure, the label of each panel indicates the offset in $p$ in units of $\delta p / 100$. In the labels, "Pxxx" corresponds to an initial precession rate $p=p_{0}+\operatorname{xxx} p / 1000$, and "Nxxx" to $p=p_{0}-\operatorname{xxx} p / 1000$. The solution "301003BIN_A.P000" is thus the nominal solution La2003. All the solutions in this figure are thus within $p_{0} \pm \delta p / 5$ and can be considered as equiprobable. They represent the variety of all the computed 1001 solutions, and some typical behavior.

In particular, we put in this plot the two solutions with closest initial conditions $p_{0} \pm \delta p / 1000$ from the nominal solution, and one can thus very well understand, by looking at the very different behavior of the three solutions "301003BIN_A.N001", "301003BIN_A.P000", "301003BIN_A.P001", that it will be very difficult to predict precisely the past evolution of Mars' obliquity over more than a few tens of millions of years. We are thus left with the only possibility; that is, to search for the possible behavior of the obliquity in the past. We will first look to the maximum possible variations of the obliquity, and then to its most probable evolution, by computing a density function for the main factors of the climate variation, the obliquity and the eccentricity. The longitude of perihelion from the moving equinox is also important for the determination of the insolation on the surface of the planet, but after a few millions of years, we can assume that it will be entirely randomized over $[0,2 \pi]$.

\subsection{Maximum variations over $250 \mathrm{Ma}$}

As we cannot display the 1001 examples that we computed, with $p \in\left[p_{0}-\delta p / 2, p_{0}+\delta p / 2\right]$, for the possible obliquity evolution over $250 \mathrm{Myr}$, we have summarized all the different cases by computing the maximum obliquity reached by the different solutions over a given prescribed time. The results are then transcribed in term of probability to reach a given obliquity before a given time (Fig. 12).

For 3, 5, or $10 \mathrm{Myr}$, the plotted curve is nearly a vertical line. This reflects the fact that, over $10 \mathrm{Myr}$, all the solutions are very close to each other, and behave in the same way. So they all reach $35^{\circ}$ before $3 \mathrm{Myr}$, remain below $40^{\circ}$ until $5 \mathrm{Myr}$, and then reach about $48^{\circ}$ before $10 \mathrm{Myr}$.

After $10 \mathrm{Myr}$, the situation is different, as due to the chaotic diffusion of the trajectories, some of them will reach high values of the obliquity. We observe that over $25 \mathrm{Myr}$, the effect of chaos is already visible, and some of

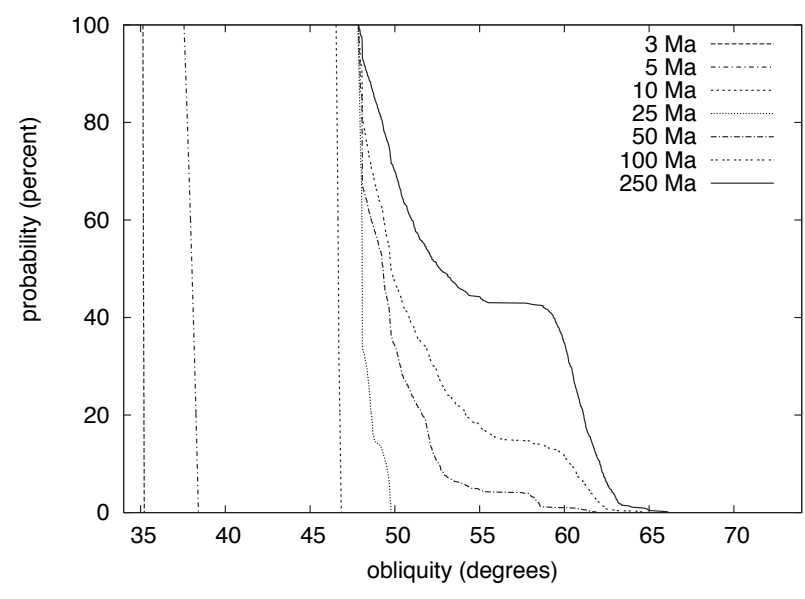

Figure 12: The probability (vertical axis) of reaching a given obliquity (horizontal axis) after a specified time is given by the different curves, for $3,5,10,25,50,100$, and 250 Myr. The estimate is obtained by 1001 integrations for regularly spaced values of $p$ ranging from $p_{0}-\delta p / 2$ arcsec/year to $p_{0}+\delta p / 2$ (Eq. 8).

the orbits reach $50^{\circ}$. After $100 \mathrm{Myr}$, a significant part of the orbits (about $13 \%$ ) went beyond $60^{\circ}$.

\subsection{The chaotic zone}

In Fig. 12, it appears that when the obliquity reaches $55^{\circ}$, then it rapidly reaches $60^{\circ}$ as well. This can be understood by looking to the shape of the chaotic zone for Mars obliquity (Fig. 13). This plot (adapted from Laskar and Robutel 1993, and Laskar 1996) is obtained using Frequency Map analysis (Laskar 1990, 1999b). In Fig. 13a, a refined determination of the precession frequency $p$ (in $\operatorname{arcsec} / \mathrm{yr}$ ) is plotted versus the initial obliquity (in degrees). It can be shown (Laskar 1999b) that regular trajectories will correspond to a smooth part of this curve, while chaotic regions will occur when the frequency curve is not regular.

The chaotic zone is divided essentially in two boxes $B 1, B 2$, that appear clearly on Fig. 13a,b. The first box $B 2$ ranges from about $10^{\circ}$ to $50^{\circ}$, while $B 1$ extents from about $30^{\circ}$ to $60^{\circ}$ (the actual size of these boxes can be measured on the ordinate axis of Fig. 13b). B1 mostly results from the secular perturbations related to the $s_{1}$ secular proper mode related to Mercury, while $B 2$ is mostly due to $s_{2}$ that is related to Venus. Each of these two modes $s_{1}, s_{2}$ is associated to multiple side terms (Fig. 13c) resulting from the complex behavior of the orbital motion of the inner Solar System, and in particular from the presence of the secular resonance associated with $\left(g_{1}-g_{5}\right)-\left(s_{1}-s_{2}\right)$ (see Laskar 1990, for more details). As a consequence for the obliquity, all the resonances with 


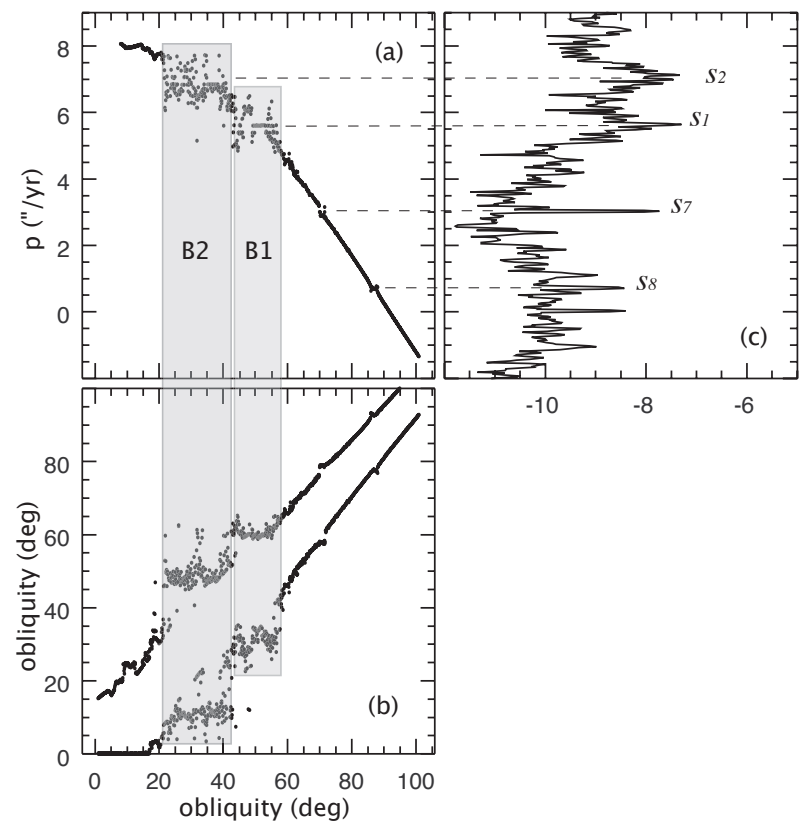

Figure 13: Frequency analysis of Mars' obliquity. (a) The frequency map is obtained by reporting in the ordinate the value of the precession frequency obtained for each of the 1000 integrations over 56 Myr for the different values of the initial obliquity (abscissa). A large chaotic zone is visible, ranging from $0^{\circ}$ to about $60^{\circ}$, with two distinct zones of large chaos, $B 1$ and $B 2$. (b) Maximum and minimum values of the obliquity reached over $56 \mathrm{Myr}$. In (c), the power spectrum of the orbital forcing term $\mathcal{A}+i \mathcal{B}$ (Eq.2) is given in logarithmic scale, showing the correspondence of the chaotic zone with the main secular frequencies $s_{1}, s_{2}, s_{7}, s_{8}$. (adapted from Laskar and Robutel 1993 and Laskar 1996).

these side terms will overlap, and thus increase the chaotic zone around $s_{1}$ and $s_{2}$. Inside $B 1$ or $B 2$, the chaotic diffusion is large, and the zone is completely explored in a relatively short time, but the communication between the two boxes is more difficult. Occasionally, a trajectory initially in $B 2$ will find the door to $B 1$, and its obliquity will reach values larger than $50^{\circ}$. But as it is now in the $B 1$ box, the strong chaotic diffusion of this box will lead the solution very rapidly to more than $60^{\circ}$. Beyond $60^{\circ}$, the frequency curve is more regular, but still not completely smooth. We can thus expect some slow diffusion to higher values of the obliquity. Moreover, the frequency map of Fig. 13a is obtained over a relatively short time. Over longer times the slow diffusion of the orbital solution itself will change slightly the values of the main frequencies (Laskar 1990), and the chaotic zones will be displaced, thus sweeping a larger area ${ }^{1}$.

\footnotetext{
${ }^{1}$ In their integrations, Touma and Widom (1993) found that Mars' obliquity only increases up to 49 degrees. This can be easily understood with the help of Fig. 13. Most probably, the solutions
}
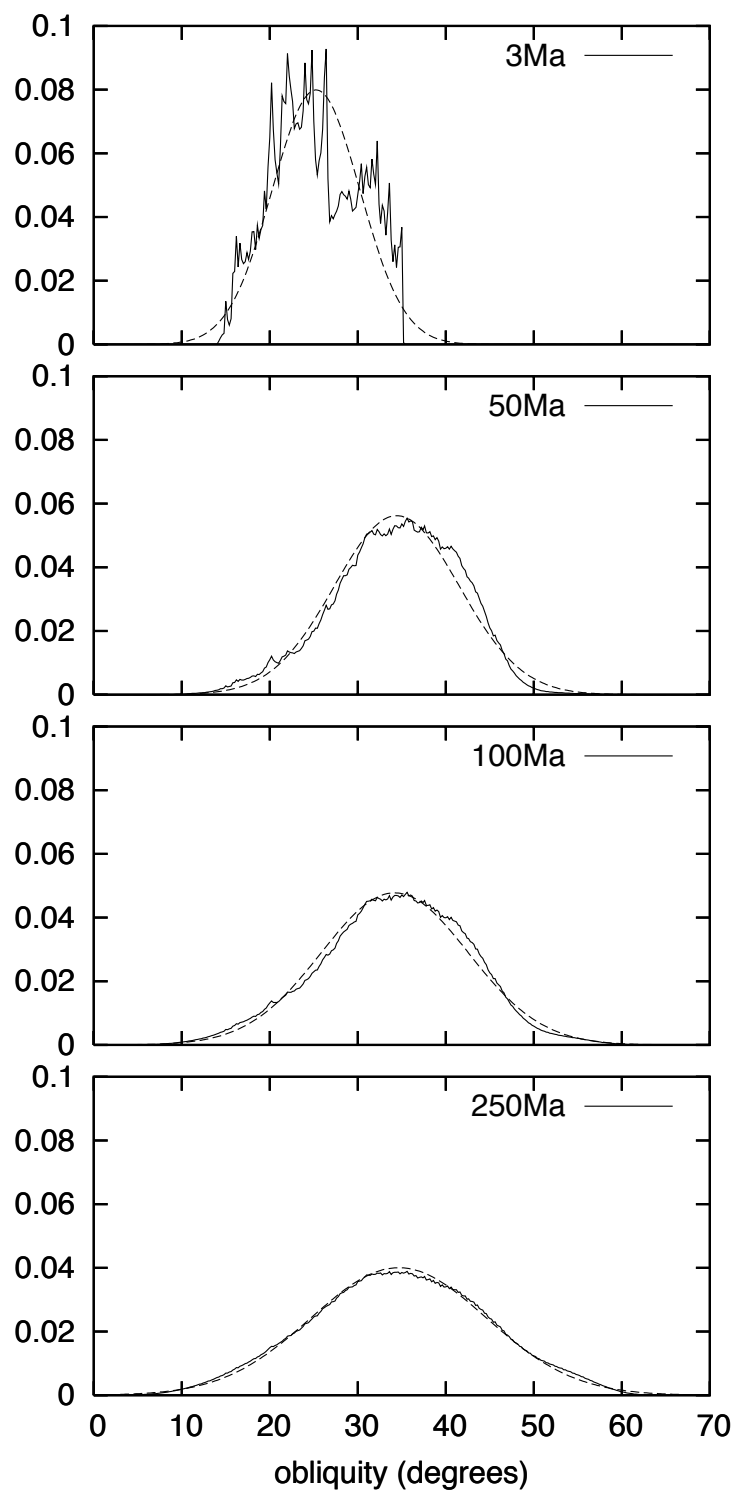

Figure 14: Normalized density function for the obliquity of Mars over 3,50, 100, and $250 \mathrm{Myr}$ (full lines). The dashed lines are the best fit with a Gaussian density.

\subsection{Obliquity statistics over $250 \mathrm{Myr}$}

The maximum value reached by the obliquity (Fig. 12) is an important quantity, as even if these extreme situations (like $\varepsilon>60^{\circ}$ ) may not last for a long time, they could induce important effects on the Martian climate evolution. But now we will look for the most probable

explored by Touma and Widom remained only in $B 2$ during their limited integration time. 
situation, by computing the density distribution of the obliquity over various time spans for all our 1001 solutions. For this purpose, we have sampled all these solutions with a sampling rate of 5000 years, and plotted the normalized density function $f_{T}(\varepsilon)$ over successive time spans $T=3,50,100,250$ Myr (Fig. 14).

It is striking to see that over relatively short time inter$\mathrm{val}$, as $3 \mathrm{Myr}$, when the chaotic behavior is not noticeable, the distribution is very irregular, but as the time interval $T$ increases, the density function $f_{T}(\varepsilon)$ becomes very smooth and very close to a pure Gaussian density $G_{m, \sigma}(x)$

$$
G_{m, \sigma}(x)=\frac{1}{\sqrt{2 \pi} \sigma} \exp \left(-\frac{(x-m)^{2}}{2 \sigma^{2}}\right)
$$

with mean value $m$ and standard deviation $\sigma$. Indeed, in Fig. 14, the obliquity distribution is plotted in full line, while we plotted in dashed line the Gaussian distribution $G_{m, \sigma}(x)$ with mean $m=\bar{\varepsilon}$ and standard deviation $\sigma=\sigma_{\varepsilon}$. The corresponding values for $\bar{\varepsilon}$ and $\sigma_{\varepsilon}$ over the various values of $T$ are given in Table 2 .

\begin{tabular}{rrrrr}
\hline$T(\mathrm{Myr})$ & $\varepsilon_{M}\left(^{\circ}\right)$ & $\varepsilon_{m}\left(^{\circ}\right)$ & $\bar{\varepsilon}\left(^{\circ}\right)$ & $\sigma_{\varepsilon}\left(^{\circ}\right)$ \\
\hline 3 & 35.218 & 15.009 & 25.254 & 4.992 \\
5 & 38.411 & 13.578 & 26.874 & 5.845 \\
10 & 46.859 & 13.578 & 31.468 & 7.187 \\
25 & 49.781 & 13.578 & 34.218 & 6.543 \\
50 & 61.903 & 5.289 & 34.427 & 7.099 \\
100 & 64.898 & 3.014 & 34.199 & 8.354 \\
250 & 66.154 & 0.043 & 34.643 & 9.966 \\
\hline
\end{tabular}

Table 2: Statistics for the obliquity evolution over 250 Myr. Maximum $\left(\varepsilon_{M}\right)$, minimum $\left(\varepsilon_{m}\right)$, average $(\bar{\varepsilon})$, and standard deviation $\left(\sigma_{\varepsilon}\right)$ of the obliquity are given over different time intervals (col. $1)$. The nominal orbital solution is used, and the statistics are performed over 1001 obliquity solutions with initial conditions for regularly spaced values of $p$ ranging from $p_{0}-\delta p / 2$ to $p_{0}+\delta p / 2$.

\section{Diffusion over 5 Gyr}

Now, we will analyze the possible evolution of the orbital and precessional solution over $5 \mathrm{Gyr}$, that is, over a time comparable to the age of the Solar System. Over this time span, we will be able better to understand the effect of the slow chaotic diffusion of the orbital motion of the Solar System.

Indeed, when the main secular frequencies of the orbital motion will change, as a result of the chaotic diffusion, the chaotic region in the frequency space (Fig. 13) will be modified, and the obliquity may enter more easily in alternate regions of the phase space. We thus expect that the diffusion of the planetary trajectories will lead to a larger diffusion of the obliquity.

\subsection{The secular equations}

In order to investigate the diffusion of the orbits over 5 Gyr, we will use the secular equations of Laskar (1990), with some small modifications. The secular equations are obtained by averaging the equations of motion over the fast angles that are the mean longitudes of the planets. Indeed, due to the degeneracy of the Keplerian problem, the other coordinate angles (longitudes of the perihelion and node) evolve much more slowly, as their motion results uniquely from the mutual planetary perturbations, and the perturbation of the pure Newtonian point mass attraction of the Sun from general relativity and quadrupole moment of the Sun. The averaging of the equation of motion is obtained by expanding the perturbations of the Keplerian orbits in Fourier series of the angles, where the coefficients themselves are expanded in series of the eccentricities and inclinations. This averaging process was conducted in a very extensive way, up to second order with respect to the masses, and through degree 5 in eccentricity and inclination, leading to truncated secular equations of the Solar System of the form
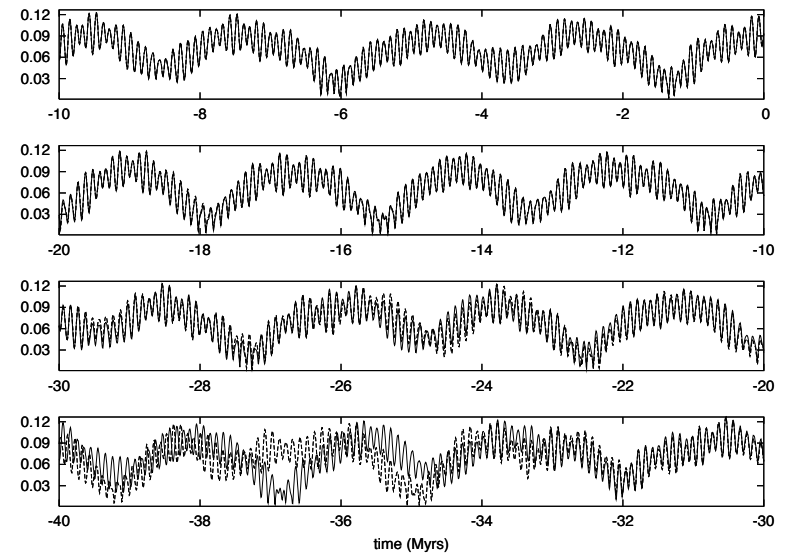

Figure 15: Eccentricity of Mars over 40 Myr. The full line is the pure numerical solution La2003, while the dotted line is obtained with the secular equations from (Laskar 1990, 1994) (Eq. 10). The two solutions are quasi-identical over 32 to $35 \mathrm{Myr}$.

$$
\frac{d w}{d t}=\sqrt{-1}\left\{\Gamma w+\Phi_{3}(w, \bar{w})+\Phi_{5}(w, \bar{w})\right\}
$$

where $w=\left(z_{1}, \ldots, z_{8}, \zeta_{1}, \ldots, \zeta_{8}\right)$, with $z_{k}=e_{k} \exp \left(\varpi_{k}\right)$, $\zeta_{k}=\sin \left(i_{k} / 2\right) \exp \left(\Omega_{k}\right)\left(\varpi_{k}\right.$ is the longitude of the perihelion). The $16 \times 16$ matrix $\Gamma$ is similar to the linear matrix that was originally derived by Lagrange and Laplace to demonstrate that at first order, the Solar System is stable, while $\Phi_{3}(w, \bar{w})$ and $\Phi_{5}(w, \bar{w})$ gather the terms of degree 3 and 5 . 


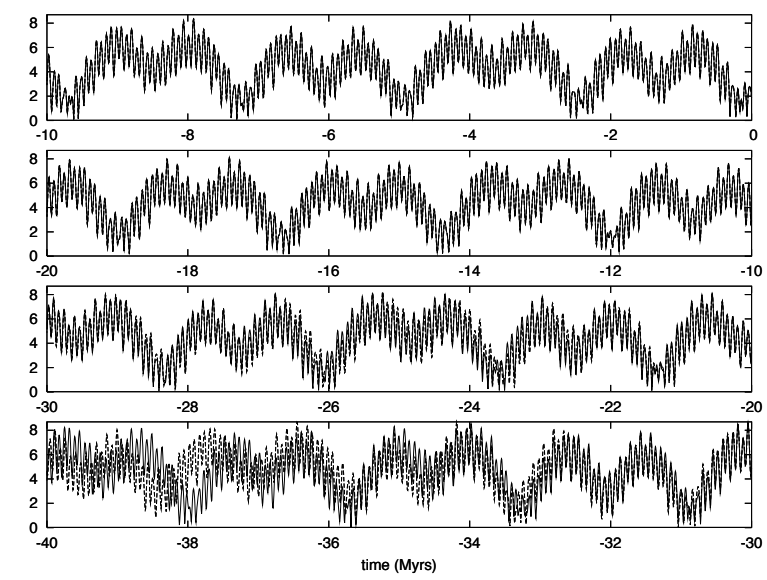

Figure 16: Inclination of Mars over 40 Myr (in degrees, referred to the ecliptic J2000). The full line is the pure numerical solution La2003, while the dotted line is obtained with the secular equations from (Laskar 1990, 1994) (Eq. 10). The two solutions are quasiidentical over 32 to 36 Myr.

The system of equations thus obtained contains some 150000 terms, but can be considered as a simplified system, as its main frequencies are now the precession frequencies of the orbits of the planets, and no longer comprise their orbital periods. The full system can thus be numerically integrated with a very large stepsize of 200 to 500 years. Contributions due to the secular perturbation of the Moon and general relativity are also included (see Laskar 1990, 1996 for more details and references).

This secular system is then simplified and reduced to about 50000 terms, after neglecting terms of very small value (Laskar 1994). Finally, a small correction of the terms of the matrix $\Gamma$ of (10), after diagonalization, is performed in order to adjust the linear frequencies, in a similar way as it was done in (Laskar 1990). Indeed, in the outer planetary system, terms of higher order are of some importance, but their effect will mainly be slightly to modify the values of the main frequencies of the system. The correction that is done here is a simple way to correct for this effect.

The original solutions of Laskar (1990) are very close to the direct numerical integration La2003 over 10 to 20 Myr (Laskar et al. 2004). With the present small adjustment, we obtain a significant improvement, and the solutions are very close over about 35 Myr (Figs. 15, 16). It should be noted that this time is also about the time over which the numerical solution itself is valid (Fig. 4), due to the imperfections of the model. Moreover, as the stepsize used in the secular equations is 200 years instead of 1.82625 days, over very long times the numerical noise will be smaller. It is thus legitimate to investigate the diffusion of the orbital motion over long times using the secular equations. The major advantage, besides reducing the roundoff errors, resides in the computation speed : the integration of the secular equations is 2000 times faster than the integration of the non-averaged equations, and we can compute a 5 Gyr solution for the Solar System in 12 hours on a Compaq alpha workstation (833 Mhz). We will thus be able to make statistics over many solutions with close initial conditions. In these computations, our main limitation will be in the huge amount of data generated by these numerical integrations.

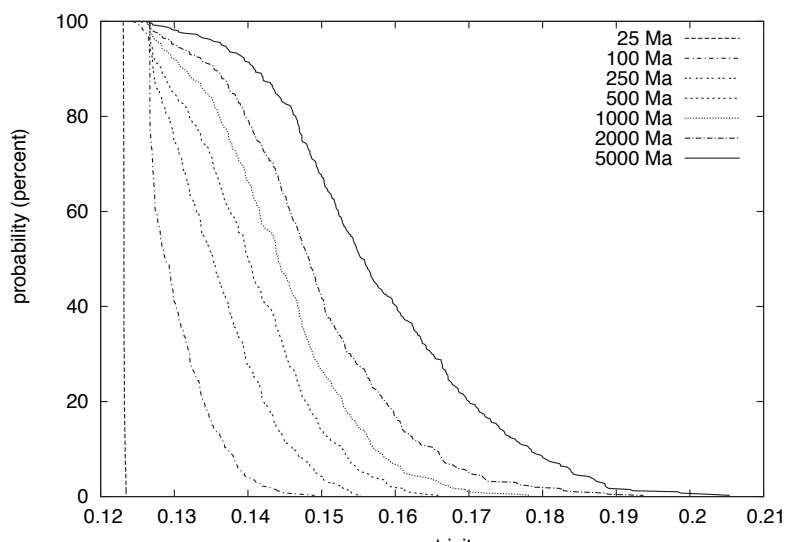

Figure 17: The probability (vertical axis) of reaching a given eccentricity (horizontal axis) after a specified time is given by the different curves, for 25, 100, 250, 500, 1000, 2000, 5000 Myr. The estimate is obtained with 403 different orbital solutions of the secular equations.

\subsection{Eccentricity}

We have integrated first 403 orbital solutions, retaining only the largest values reached by the eccentricity. The results of the maximum eccentricity reached after 25,100 , 250, 500, 1000, 2000, and $5000 \mathrm{Myr}$ are given in Fig. 17. As expected in a diffusive process, the maximum value reached by the eccentricity increases slowly with time.

We have also performed 200 orbital solutions, retaining the values of the eccentricity with a sampling rate of 25000 years in order to establish the density distribution of the eccentricity over different time spans (Fig. 18). It is striking that the density function converges towards a very smooth density function beyond $1 \mathrm{Gyr}$, as the chaotic diffusion is dominant, while over $25 \mathrm{Myr}$, where the motion is very predictable, the density distribution cannot be approximated by a simple function. The associated mean values $(\bar{e})$ and standard deviations $\left(\sigma_{e}\right)$ of the eccentricity distributions are given in Table 3 . 

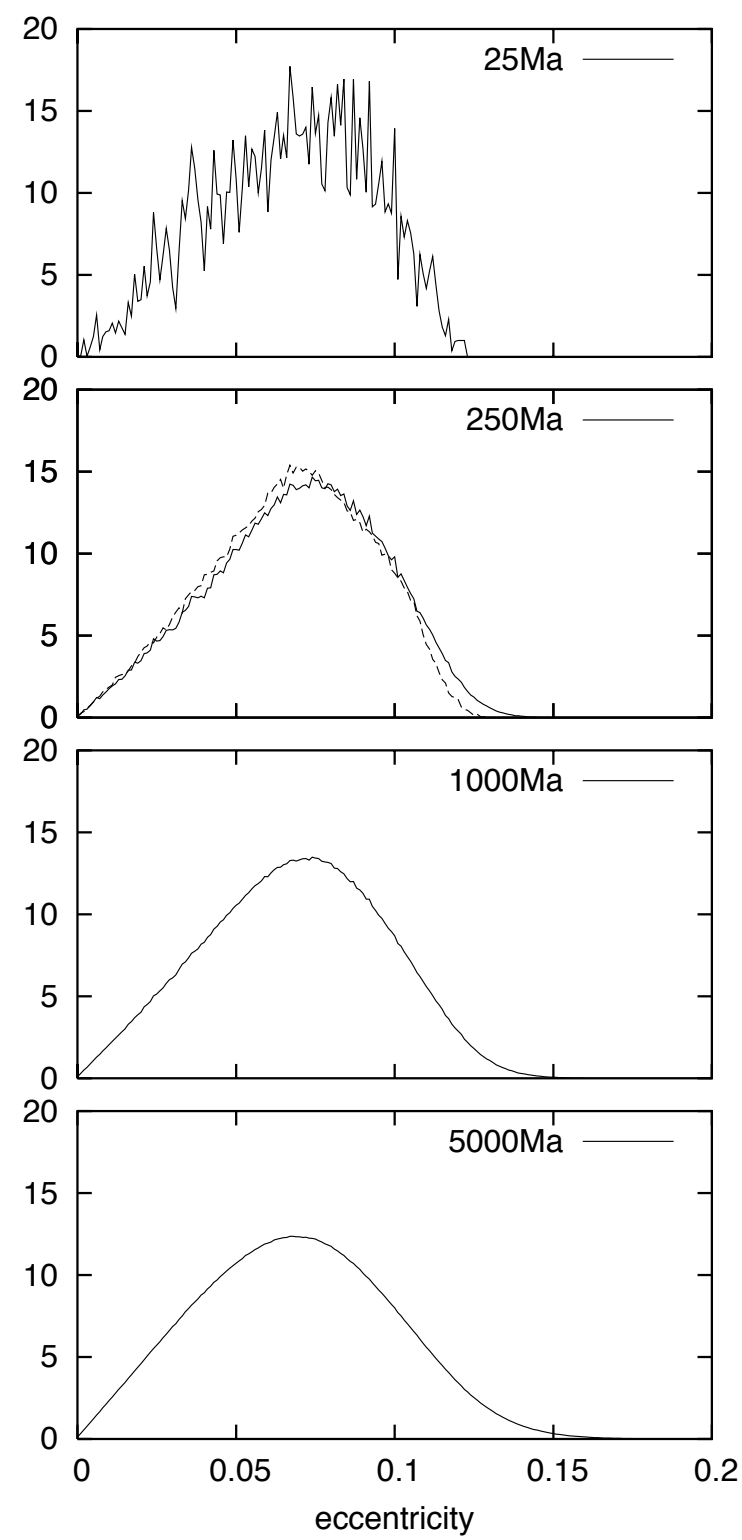

Figure 18: Normalized density function for the eccentricity of Mars over 25, 250, 1000, and $5000 \mathrm{Myr}$. For $250 \mathrm{Myr}$, the dotted line is the density obtained over the pure numerical solution La2003.

In Fig. 18, even if the density function of the eccentricity over $5000 \mathrm{Gyr}$ is very smooth, it appears to be slightly different from a true Gaussian function. In particular, the density goes to zero for zero eccentricity, with a linear behavior near the origin. This will be discussed in section 5.4.1.

\begin{tabular}{rrr}
\hline$T(\mathrm{Myr})$ & $\bar{e}$ & $\sigma_{e}$ \\
\hline 25 & 0.066765 & 0.025551 \\
100 & 0.069046 & 0.025592 \\
250 & 0.069664 & 0.026361 \\
500 & 0.069430 & 0.026914 \\
1000 & 0.068936 & 0.027725 \\
2000 & 0.068754 & 0.028602 \\
5000 & 0.068989 & 0.029937
\end{tabular}

Table 3: Statistics for the eccentricity evolution over 5 Gyr. 400 solutions of the secular equations are integrated over 5 Gyr with close initial conditions.

\subsection{Obliquity}

We have done the same analysis for the obliquity over 5 Gyr, with 1001 solutions of the obliquity, for each of the 200 orbital solutions, integrated with close initial conditions. The maximum obliquity reached after 10, 25, 50, $100,250,500,1000,2000,3000,5000 \mathrm{Myr}$ are given in Tables 4 and 5 and in Fig. 19.

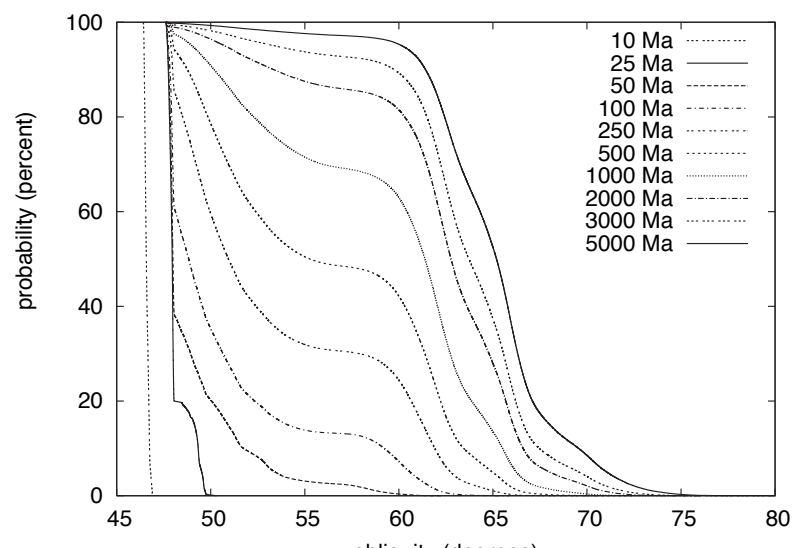

Figure 19: The probability (vertical axilisuity (degrees) of reaching a given obliquity (horizontal axis) after a specified time if given by the different curves, for 10, 25, 100, 250, 500, 1000, 2000, 3000, 5000 Myr. The estimate is obtained with 200 different orbital solutions, with, for each of them, 1001 integrations for regularly spaced values of $p$ ranging from $p_{0}-\delta p / 2$ to $p_{0}+\delta p / 2$.

The density function for the obliquity over 50, 100, 250, 500, 1000, 2000, 3000, $4000 \mathrm{Myr}$ is displayed in Fig. 20, and the corresponding mean values $\bar{\varepsilon}$ and standard deviation $\sigma_{\varepsilon}$ are given in Table 6 .

The maximal value reached by the obliquity is $82.035^{\circ}$, although the probability to reach more than $80^{\circ}$ over 5 Gyr is only $0.015 \%$ (Table 5). More realistically, there are still $4 \%$ of the solutions that went beyond $70^{\circ}$ in less 


\begin{tabular}{rrr}
\hline$T(\mathrm{Myr})$ & $\varepsilon_{M}\left(^{\circ}\right)$ & $\varepsilon_{m}\left(^{\circ}\right)$ \\
\hline 5 & 38.442 & 13.594 \\
10 & 46.907 & 13.594 \\
25 & 50.310 & 13.594 \\
50 & 62.274 & 3.786 \\
100 & 65.874 & 0.014 \\
250 & 70.508 & 0.001 \\
500 & 72.543 & 0.001 \\
1000 & 74.678 & 0.000 \\
2000 & 76.005 & 0.000 \\
3000 & 79.465 & 0.000 \\
5000 & 82.035 & 0.000 \\
\hline
\end{tabular}

Table 4: Maximal and minimal values reached by the obliquity over 5 Gyr. 200 solutions of the secular equations were integrated over 5 Gyr with close initial conditions, and for each orbital solution, 1001 obliquity solutions with initial conditions for regularly spaced values of $p$ ranging from $p_{0}-\delta p / 2$ to $p_{0}+\delta p / 2$ were integrated.

\begin{tabular}{rrrrrr}
\hline$T$ (Myr) & $60^{\circ}$ & $65^{\circ}$ & $70^{\circ}$ & $75^{\circ}$ & $80^{\circ}$ \\
\hline 5000 & 95.35 & 52.51 & 8.51 & 0.270 & 0.015 \\
3000 & 89.29 & 37.38 & 4.07 & 0.025 & \\
2000 & 81.47 & 27.94 & 2.08 & 0.005 & \\
1000 & 63.00 & 13.52 & 0.36 & & \\
500 & 42.16 & 4.92 & 0.03 & & \\
250 & 24.40 & 1.03 & 0.01 & & \\
100 & 7.23 & & & & \\
50 & 0.36 & & & & \\
25 & & & & & \\
\hline
\end{tabular}

Table 5: Numerical examples taken from the data of Fig. 19. For each time span $T$, we give respectively the probability (in percent) for the obliquity $\varepsilon$ to reach $60^{\circ}, 65^{\circ}, 70^{\circ}, 75^{\circ}$, or $80^{\circ}$. When there is no occurrence of the event, the corresponding space is left blank.

than 3 Gyr. In these cases, the orbits were able to find their way in the zone of chaotic small diffusion between the resonances $s_{1}$ and $s_{7}$ (Fig. 13). Finally, we are nearly certain that the obliquity of Mars went to higher values than $60^{\circ}$ during its history, as $95 \%$ of the solutions reached this value. Closer to the present, the probability to have reached $60^{\circ}$ before 1 Gyr is about $63 \%$.

In Fig. 21, the similar quantities are plotted for a single solution of the obliquity. In this case, the density function of the obliquity is very different, as over 4 Gyr (full line), it has two distinct maxima. Indeed, the individual solutions can present very different behavior, and in the present case, it appears that the solution remained for a long time in the first "box" $B 1$ related to secular resonances with $s_{1}$, and also for a long period in the "box" $B 2$ related to $s_{2}$, but never much in between (see Fig. 13). When the statistics are made over many orbital solutions, these regions will be displaced, and the repartition of the obliquity will become more even.

\begin{tabular}{rrr}
\hline$T(\mathrm{Myr})$ & $\bar{\varepsilon}\left(^{\circ}\right)$ & $\sigma_{\varepsilon}\left(^{\circ}\right)$ \\
\hline 50 & 34.244 & 7.149 \\
100 & 34.068 & 8.438 \\
250 & 34.432 & 9.979 \\
500 & 35.082 & 10.977 \\
1000 & 35.950 & 11.987 \\
2000 & 36.779 & 12.978 \\
3000 & 37.267 & 13.499 \\
4000 & 37.620 & 13.814 \\
\hline
\end{tabular}

Table 6: Mean value $(\bar{\varepsilon})$ and standard deviation $\left(\sigma_{\varepsilon}\right)$ for the the obliquity over $50 \mathrm{Myr}$ to 4 Gyr. 19 solutions of the secular equations were integrated over 4 Gyr with close initial conditions, and for each orbital solution, 501 obliquity solutions with initial conditions for regularly spaced values of $p$ ranging from $p_{0}-\delta p / 2$ to $p_{0}+\delta p / 2$ were integrated.

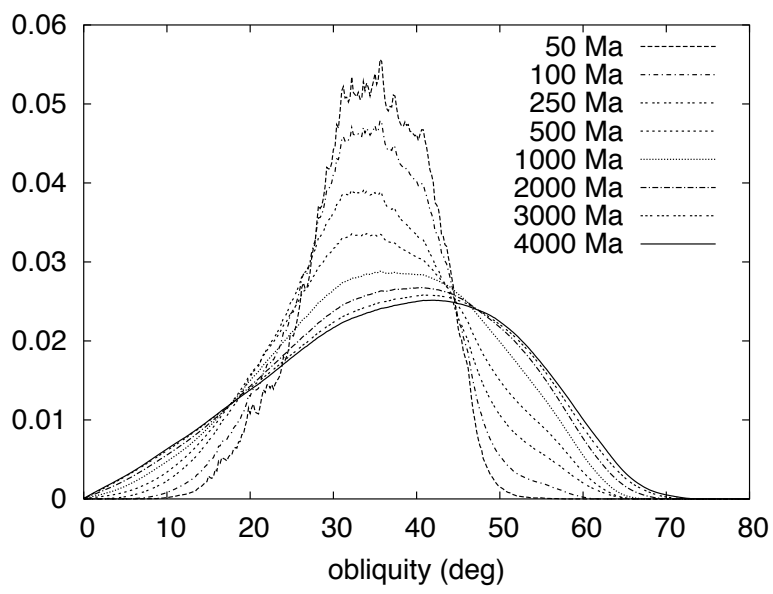

Figure 20: Normalized density distribution of the obliquity of Mars, over 50,100, 250, 500, 1000, 2000, 3000, and $4000 \mathrm{Myr}$. This is obtained with 19 different orbital solutions, and for each of them 501 obliquity solutions, with a sampling time of 1000 years.

\subsection{Diffusion laws}

We have seen that the density functions of the eccentricity (Fig. 18) or obliquity (Fig. 20) over 5 Gyr are smooth functions, that evolve with time and differ significantly from pure Gaussian distributions. In this section, we will look to the evolution with time of these distributions, and analyze the diffusion of the solutions over time. The goal is to establish some simple formulas that will allow to represent the distributions of the eccentricity and obliquity over very long times, of several Gyr. Quite remarkably, although it is impossible to predict the precise evolution of the individual trajectories, we will be able to give very simple expressions that fit very well with the observed eccentricity and obliquity distributions. 


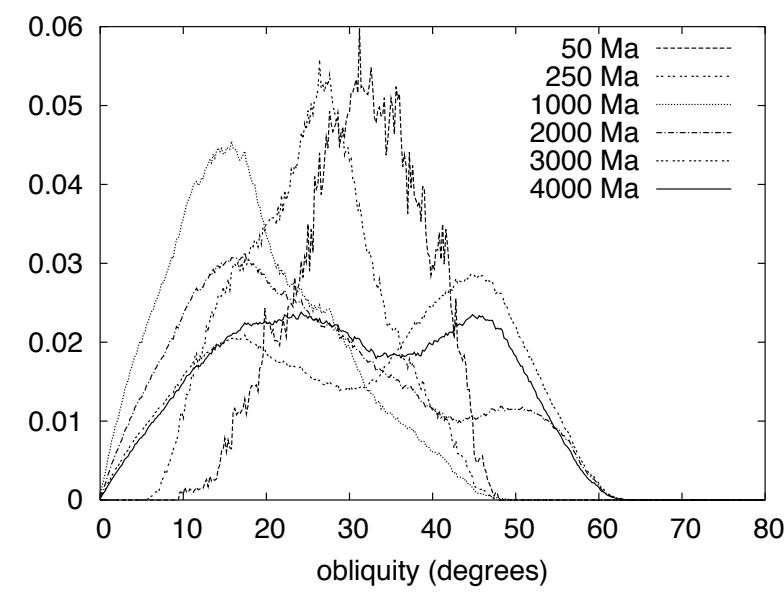

Figure 21: Normalized density distribution of the obliquity of Mars, over 50, 250, 1000, 2000, 3000, and $4000 \mathrm{Myr}$. This is obtained with a single solution with a sampling time of 1000 years.

\subsubsection{Eccentricity}

We first have looked at the evolution of the mean value of the eccentricity $\bar{e}$ and its standard deviation $\sigma_{e}$ over $5 \mathrm{Gyr}$, computing these quantities over 20 consecutive intervals of $250 \mathrm{Myr}$ (Fig. 22). The mean value $\bar{e}$ does not show significant evolution, but the standard deviation increases steadily and is well fitted with

$$
\sigma_{e}=0.028678+0.001676 \log (T)
$$

where the time $T$ is expressed in Gyr. In a pure Brownian motion, the diffusion speed $d \sigma^{2} / d t$ is constant, while here, when neglecting the terms in $\log ^{2}(T)$ that are small over a few Gyrs, we have

$$
\frac{d \sigma_{e}^{2}}{d T} \propto \frac{1}{T}
$$

The diffusion rate becomes thus very slow as $T$ increases.

At the moment, we have no possibility to derive analytically the observed density function from the equation of motion, but we have searched for the best possible fit with a small number of parameters (Fig. 23). As was already observed, the Gaussian density is not a good candidate as the observed density goes to zero when the eccentricity goes to zero, with a non-zero derivative. In fact, a model that fits very well with the density of the eccentricity is given by a one-dimensional random walk with an absorbing edge at zero (see for example Grimmett and Stirzaker
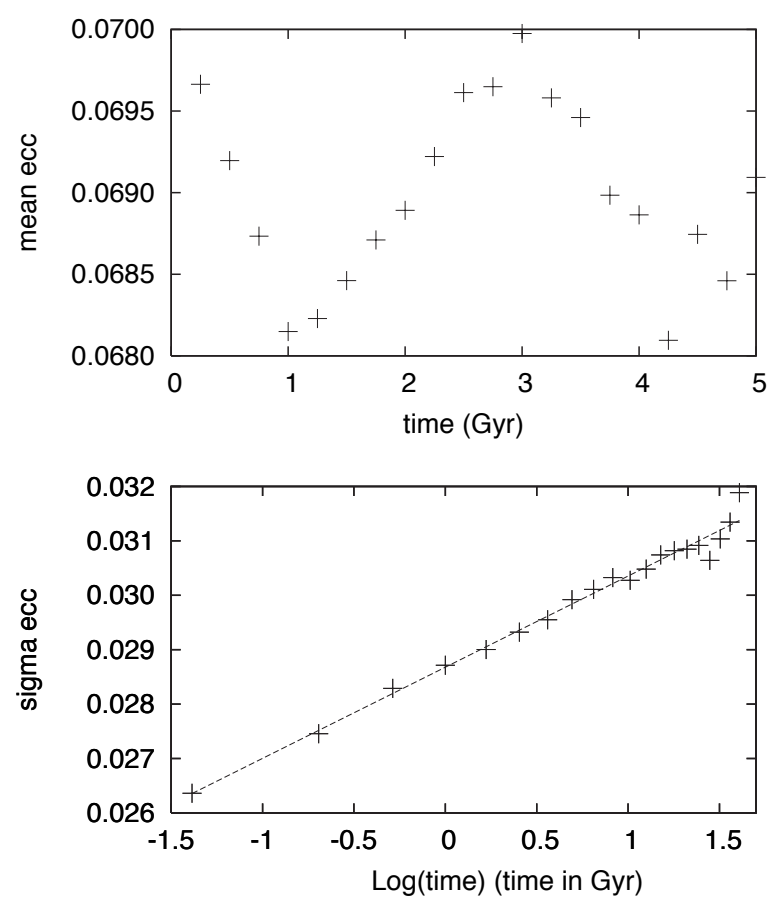

Figure 22: Evolution of the mean value $\bar{e}$ (top) and standard deviation $\sigma_{e}$ (bottom) for the eccentricity of Mars. The statistics are made over 20 consecutive intervals of $250 \mathrm{Myr}$, for 200 orbits with close initial conditions. The standard deviation is fitted with the line $\sigma_{e}=0.028678+0.001676 \log (T)$ where the time $T$ is in Gyr.

2001). For such a simple model, the density function is

$$
\tilde{G}_{\tilde{m}, \tilde{\sigma}}(x)=\frac{\exp \left(-\frac{(x-\tilde{m})^{2}}{2 \tilde{\sigma}^{2}}\right)-\exp \left(-\frac{(x+\tilde{m})^{2}}{2 \tilde{\sigma}^{2}}\right)}{\sqrt{2 \pi} \tilde{\sigma} \operatorname{erf}\left(\frac{\tilde{m}}{\sqrt{2} \tilde{\sigma}}\right)}
$$

where $\operatorname{erf}(x)$ is the error function

$$
\operatorname{erf}(x)=\frac{2}{\sqrt{\pi}} \int_{0}^{x} e^{-t^{2}} d t
$$

In Fig. 23, the density function $f_{e}(x)$ of the eccentricity on the time interval $[4.75 \mathrm{Gyr}, 5 \mathrm{Gyr}]$ is plotted in full line, while the dashed line is the best fit with a Gaussian density. Barely visible, as it superposes nearly exactly with the filled curve, is the best least square approximation of $f_{e}(x)$ with $\tilde{G}_{\tilde{m}, \tilde{\sigma}}(x)$, with $\tilde{m}=0.063831$ and $\tilde{\sigma}=0.037436$. It should be noted that the mean $m_{a}$ and standard deviation $\sigma_{a}$ for the density function $\tilde{G}_{\tilde{m}, \tilde{\sigma}}(x)$ 
are not $\tilde{m}$ and $\tilde{\sigma}$, but slightly different :

$$
m_{a}=\frac{\tilde{m}}{\operatorname{erf}\left(\frac{\tilde{m}}{\sqrt{2} \tilde{\sigma}}\right)}
$$

and

$$
\sigma_{a}^{2}=\tilde{\sigma}^{2}+\tilde{m}^{2}-m_{a}^{2}+\sqrt{\frac{2}{\pi}} \tilde{\sigma} m_{a} \exp \left(-\frac{\tilde{m}^{2}}{2 \tilde{\sigma}^{2}}\right) .
$$

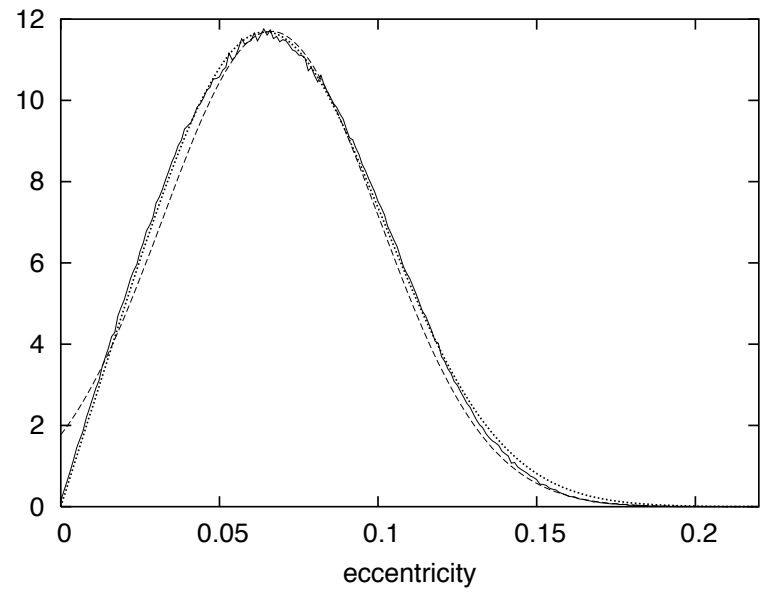

Figure 23: Normalized density function for the eccentricity of Mars on the time interval $[4.75,5]$ Gyr (full line). The dashed line is the best fit Gaussian distribution, and the dotted line is the best fit with a distribution of a random walk with an absorbing end at 0 (Eq. 13).

As this density $\tilde{G}_{\tilde{m}, \tilde{\sigma}}(x)$ represents very precisely the evolution of Mars' eccentricity from 500 Myr to 5 Gyr, we have computed the corresponding parameters $\tilde{m}, \tilde{\sigma}$ (Fig. $24)$ that can be approximated over $[0.5 \mathrm{Gyr}, 5 \mathrm{Gyr}]$ by

$$
\begin{aligned}
& \tilde{m}=0.069367-0.001109 T \\
& \tilde{\sigma}=0.032131+0.002641 \log (T),
\end{aligned}
$$

where the time $T$ is in Gyr. In some sense, the relations (13) and (17) provide a good predictive model for the eccentricity of Mars beyond 500 Myr.

\subsubsection{Obliquity}

We have performed the same study for Mars' obliquity. In this case, the analysis was performed for 19 orbital solutions, and for each of them 500 obliquity solutions with close initial conditions. The statistics were made over 40 consecutive intervals of $100 \mathrm{Myr}$. Contrarily to the eccentricity, the mean obliquity $\bar{\varepsilon}$ presents a slow increase
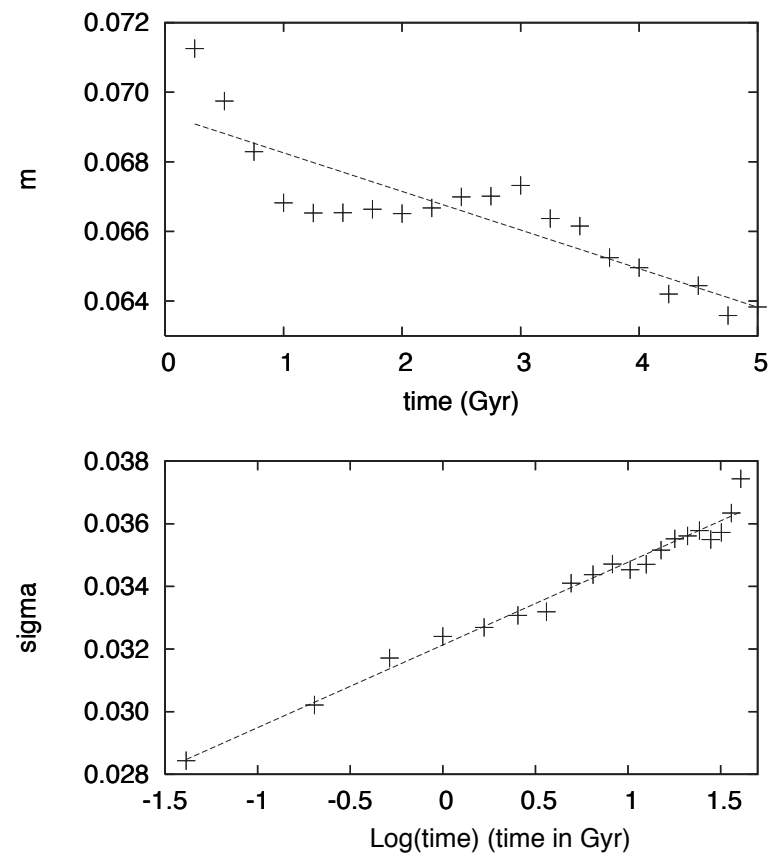

Figure 24: Evolution with time of the parameters $\tilde{m}$ (top) and $\tilde{\sigma}$ (bottom) of the eccentricity density distribution given in (Eq. 13). The linear fits are made respectively with $\tilde{m}=0.069367-0.001109 T$ and $\tilde{\sigma}=0.032131+0.002641 \log (T)$, where the time $T$ is in Gyr.

with time as well as its standard deviation that can be well approximated (Fig.25) with

$$
\begin{aligned}
& \bar{\varepsilon}=37.007+1.345 \log (T) \\
& \sigma_{\varepsilon}=13.301+1.426 \log (T)-0.259 \log ^{2}(T)
\end{aligned}
$$

The diffusion of the obliquity thus follows a similar law as (12) for the eccentricity. In a similar way as with the eccentricity, we have plotted the last density function $f_{40}(\varepsilon)$, obtained over the interval [3.9 Gyr, 4.0 Gyr] (Fig. 27 , full line). The density $f_{40}(\varepsilon)$ is also far from a Gaussian density (dashed line), although the density $f_{1}(\varepsilon)$ for [0 Myr, $100 \mathrm{Myr}]$ is very similar to a Gaussian density. In fact the obliquity density of (Fig. 27) is also different from the density of the eccentricity (Eq. 13). After some trials, we found a very good fit of $f_{40}(\varepsilon)$ with a sine function on $\left[0^{\circ}, 40^{\circ}\right]$. This led us to search for the density function $f_{Y}$ of $Y=1-\cos (\varepsilon)$ instead of $\varepsilon$ (Fig. 26). We have found a very good agreement of the observed density $f_{Y}$ with

$$
f_{m, b}(x)=\frac{1}{2 m}\{\operatorname{erf}(b(x+m))-\operatorname{erf}(b(x-m))\} .
$$

This agreement is in fact so precise that the two curves are barely distinguishable on Fig. 26. The density $f_{m, b}(x)$ 

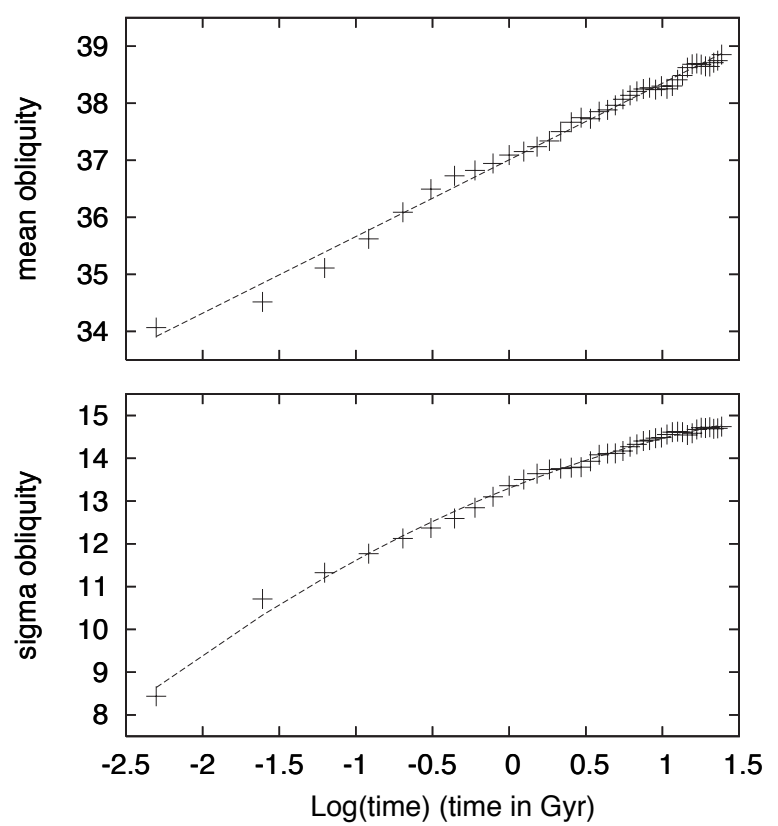

Figure 25: Evolution of the mean value $\bar{\varepsilon}$ (top) and standard deviation $\sigma_{\varepsilon}$ (bottom) for the obliquity of Mars. The statistics are made over 40 consecutive intervals of $100 \mathrm{Myr}$, for about 10000 orbits with close initial conditions. The mean obliquity is fitted with $\bar{\varepsilon}=37.007+1.345 \log (T)$, while the standard deviation is fitted with $\sigma_{\varepsilon}=13.301+1.426 \log (T)-0.259 \log ^{2}(T)$ where $T$ is in Gyr.

is in fact the density of $|X+Y|$, where $X, Y$ are two independent random variables. The random variable $X$ has a uniform density on $[-m, m]$, and $Y$ is a centered Gaussian variable with standard deviation

$$
\sigma=\frac{1}{\sqrt{2} b} .
$$

In fact, $f_{m, b}(x)$ is a density on $[0,+\infty[$, and not on $[0,2]$. Nevertheless, for the values of $b, \hat{m}$ that we will use $(b \approx 8, \hat{m} \approx 0.5)$, and for $x>2$,

$$
f_{m, b}(x) \simeq \frac{2 b}{\sqrt{\pi}} \exp \left(-b^{2} x^{2}\right),
$$

and

$$
\int_{0}^{2} f_{\hat{m}, b}(x) d x \simeq \operatorname{erf}(2 b) .
$$

For $b=8,1-\operatorname{erf}(2 b) \simeq 0.23 \times 10^{-112}$. We can thus consider, for our practical needs, that $f_{m, b}(x)$ is a density on $[0,2]$. Once we have a good candidate for the density of $1-\cos (\varepsilon)$, we obtain easily the density of $\varepsilon$ on $[0, \pi]$ as

$$
\begin{aligned}
\hat{G}_{\hat{m}, b}(x)=\frac{\sin (x)}{2 \hat{m}} & \{\operatorname{erf}(b[1-\cos (x)+\hat{m}]) \\
& -\operatorname{erf}(b[1-\cos (x)-\hat{m}])\} .
\end{aligned}
$$

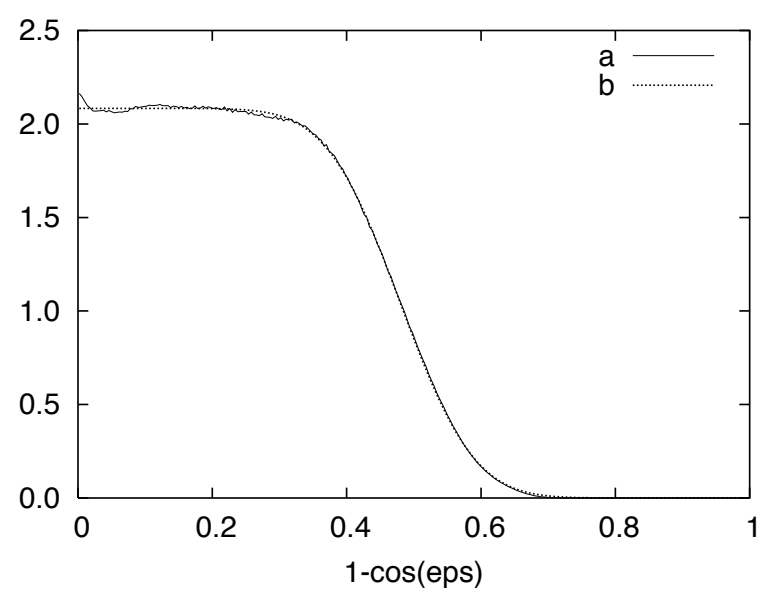

Figure 26: Normalized density function for $1-\cos (\varepsilon)$ (full line, (a)), where $\varepsilon$ is the obliquity of Mars on the time interval $[3.9,4]$ Gyr. The dotted line (b) the best fit with the density function given in Eq. (19).

The parameters $\hat{m}, b$ are determined by least square fit to the observed density of the obliquity, and we find $\hat{m}_{40} \simeq 0.479, b_{40} \simeq 7.793$. As with the eccentricity, the agreement is so good that $\hat{G}_{\hat{m}, b}(x)$, plotted with a dotted line (label c ) in Fig. 27 is barely discernible from the density of the obliquity.

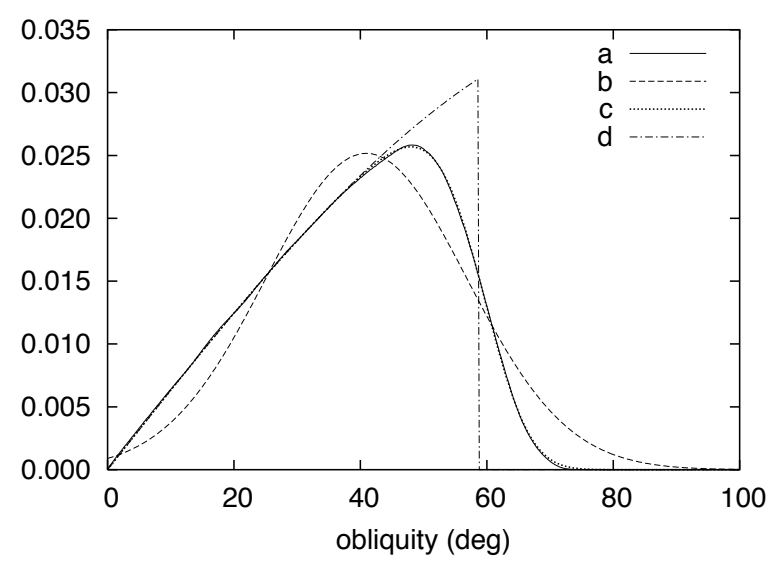

Figure 27: Normalized density function for the obliquity $(\varepsilon)$ of Mars on the time interval $[3.9,4]$ Gyr (full line, (a)). The dashed line (b) is the best fit Gaussian distribution, and the dotted line (c) the best fit with the density function given in (Eq. 23). The curve (d) is the density (26) of the uniform probability on the spherical cap limited by $\varepsilon=\varepsilon_{\tilde{m}}$.

We have then determined the parameters $\hat{m}, b$ on each time interval, but instead of plotting $\hat{m}$ in Fig. 28, we have preferred to display the corresponding value of the 
obliquity $\left(\varepsilon_{\hat{m}} \simeq 58.622^{\circ}\right.$ in Fig. 27$)$

$$
\varepsilon_{\hat{m}}=\arccos (1-\hat{m}) \text {. }
$$

The value of $b$ is nearly constant, with mean value $\bar{b}=$ 7.825 , while $\varepsilon_{\hat{m}}$ can be approximated by

$$
\varepsilon_{\hat{m}}=54.515+3.726 \log (T)-0.583 \log ^{2}(T)
$$

where $T$ is in Gyr. As for the eccentricity, Eqs. (23) and (25) provide a precise description of the obliquity evolution beyond $500 \mathrm{Myr}$. It should be noted that the
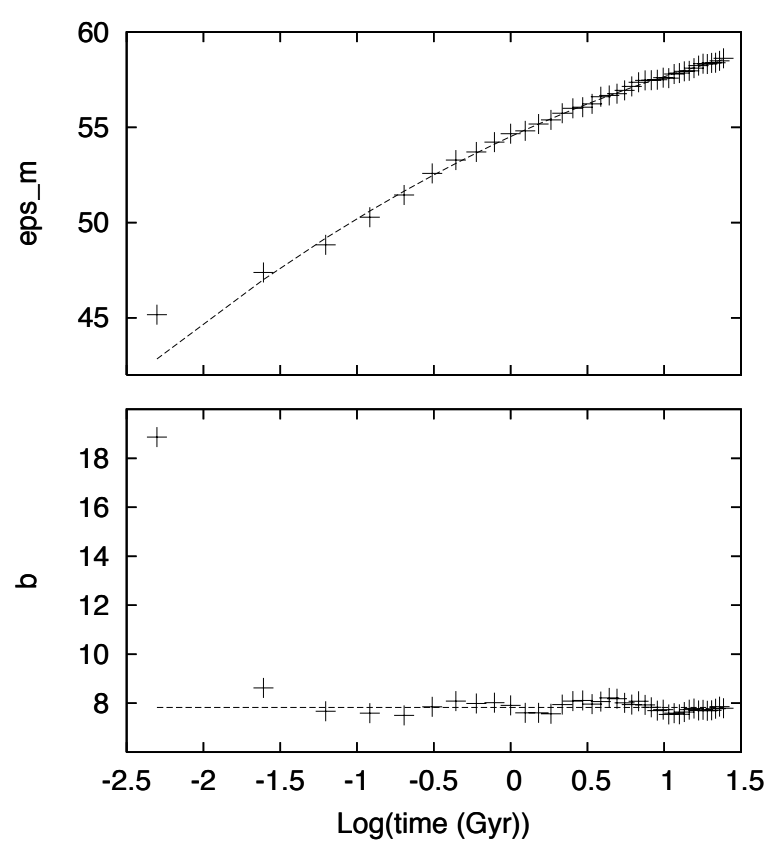

Figure 28: Evolution with time of the parameters $\tilde{m}$ (top) and $b$ (bottom) of the obliquity density distribution given in Eq. (23). The least square fit of $\tilde{m}$ is $\tilde{m}=54.515+3.726 \log (T)-0.583 \log ^{2}(T)$. The mean value of $b, b_{m}=7.825$ is plotted in dotted line.

density (Fig. 27d)

$$
f_{\hat{m}}(x)=\frac{\sin (x)}{\hat{m}} 1_{\left[0, \varepsilon_{\hat{m}]}\right]}(x),
$$

where $1_{\left[0, \varepsilon_{\hat{m}}\right]}(x)$ is the characteristic function of the interval $\left[0, \varepsilon_{\hat{m}}\right]$, represents also the uniform density on a spherical cap centered on the normal to the orbital plane of Mars, and limited by $\varepsilon=\varepsilon_{\hat{m}}$, with the usual Lebesgue measure on the sphere.

We can now look back to the evolution of the density with time given in Figs. 14 and 20. Over a short time (50 Myr), the obliquity is localized around a mean value of about $34^{\circ}$, with a Gaussian density; but as the diffusion process evolves, the axis density function will evolve towards a uniform distribution on the spherical cap limited by $\varepsilon=\varepsilon_{\hat{m}}$, where $\varepsilon_{\hat{m}}$ is given by Eq. (25) or Fig. 28, with an additional random noise. The distribution of the action variable $X=L \cos \varepsilon$, or equivalently of the precession frequency $(\propto \cos \varepsilon)$, will also be uniform with the addition of a random noise.

\section{Conclusions}

The new orbital and obliquity solution presented here can be used over 10 to $20 \mathrm{Myr}$ for precise paleoclimate studies on Mars. It is important to recall that the severe increase of obliquity at $5 \mathrm{Myr}$ is robust with respect to the uncertainty on the model and initial conditions. It thus provides a constraint on the possible past evolution of the Martian climates, and in particular on the evolution of the ice caps (Laskar et al. 2002). The full solution, together with subroutines allowing the computation of the insolation for various latitudes, derived from the similar routines used for the Earth paleoclimate studies (Laskar et al. 1993) is available on the WEB at http://www.imcce.fr/Equipes/ASD/mars.html.

The orbital solution of Mars should be precise over about $40 \mathrm{Myr}$, much longer than the time of validity of the obliquity and precession quantities. We have provided as well the nominal orbital solution of Mars over 100 Myrs, for reference, and as an example of possible evolution, although we know that with the problem of numerical roundoff error alone, the solution has practically no chance to be valid over more than $60 \mathrm{Myr}$.

Over longer time scale, beyond $100 \mathrm{Myr}$, the chaotic regime prevails, and we cannot give any precise evolution of the obliquity or of the orbit. Nevertheless, and quite surprisingly, we are able to give here a very precise estimate of the density function of the obliquity and eccentricity over the age of the Solar System, and have thus in a very concise way all the information for producing statistical estimates of the past climate evolution of Mars along its history. In particular, beyond $500 \mathrm{Myr}$, the density distribution of the axis of Mars tends towards a uniform density on a spherical cap limited by $\varepsilon=\varepsilon_{\hat{m}}$ (Eq. $25)$, with the addition of a random noise that produces a slow diffusion of the obliquity beyond $\varepsilon_{\hat{m}}$.

The computations beyond $250 \mathrm{Myr}$ are made with the averaged equations of Laskar (1990), but this should not modify significantly the results from a similar study made with non averaged equations as the two solutions are very similar over 35 Myr (Figs. 15 and 16), the chaotic behavior of the inner planets mostly results from their secular interactions (Laskar 1990), and the statistics made over the overlapping $250 \mathrm{Myr}$ on the obliquity are very similar 
(Tables 2 and 6).

It is remarkable that the present value of the obliquity of Mars $\left(\varepsilon_{0} \approx 25.19^{\circ}\right)$ is very far from its mean value, evaluated over $4 \mathrm{Gyr}\left(\bar{\varepsilon} \approx 37.62^{\circ}\right)$; it is even further from its most probable value over $4 \mathrm{Gyr}, \varepsilon_{s} \approx 41.80^{\circ}$ obtained for the maximum of the $4 \mathrm{Gyr}$ density of Fig. 20. The situation is different for the eccentricity, as the mean $(\bar{e} \approx$ $0.069)$ and most probable value $\left(e_{s} \approx 0.068\right)$ are smaller than the present value $\left(e_{0} \approx 0.093\right)$. In the search of the past climatic evolution of Mars, one could thus define the "standard model" of Mars as the most probable one, with $e_{s}=0.068, \varepsilon_{s}=41.80^{\circ}$.

This suggests that the present dry and cold Martian climate is probably not representative of the past current environmental conditions. Climatic simulations with orbital parameters close to the "standard model" shows that large increases in summer insolation may cause dynamical instability of the polar caps and an intensive sublimation multiplying global atmospheric humidity by a factor 50 (Jakosky et al. 1995, Richardson and Wilson 2002, Mischna et al. 2003). If not limited by a thermally protecting dust lag, dramatic annual water loss (estimated close to $10 \mathrm{~cm} / \mathrm{yr}$ ) would lead to a quick disappearance of polar caps over some obliquity cycles and to deposition of stable surface ice in equatorial areas (Jakosky et al. 1995). Geomorphological features should illustrate this now "current" latter situation. We can expect that conjugated efforts in climate modelling (e.g. Haberle et al. 2001, Mischna et al. 2003), geomorphological observations from present and future spacecraft missions (e.g. Head and Marchant 2003) and comparison with astronomical solutions will improve the reconstruction of the past "high obliquity" Martian climate.

\section{Acknowledgments}

J. L. thanks A. Coquio and B. Derrida for discussions on random processes. A large part of the computations were made at IDRIS-CNRS, and we benefited from support from PNP-CNRS, and CS from Paris Observatory.

\section{References}

Acuña, M.H., Connerney, J.E.P., Ness, N.F., Lin, R.P., Mitchell, D., Carlson, C.W., McFadden, J., Anderson, K.A., Rème, H., Mazelle, C., Vignes, D., Wasilewski, P., and Cloutier, P. 1999. Global distribution of crustal magnetization discovered by the Mars Global Surveyor MAG/ER Experiment. Science 284, 790-793.
Baker, V.R., Strom, R.G., Gulick, V.C., Kargel, J.S., Komatsu, G. and Kale, V.S. 1991. Ancient oceans, ice sheets and the hydrological cycle on Mars. Nature 352, 589-594.

Baker, V.R. 2001. Water and the Martian landscape. Nature 412, 228-236.

Barriot J.-P., Dehant V., Cerisier J.-C., Folkner W., Rhibes A., Benoist J., T. Van Hoolst, R. Warnant, R.A. Preston, L. Romans, S. Wu, and A.W. Wernik 2001. NEIGE: NEtlander Ionosphere and Geodesy Experiment. Adv. Space Res. 28(8), 1237-1249.

Bills, B.G. 1999. Obliquity-oblateness feedback on Mars. J. Geophys. Res. 104, 30773-30797.

Bretagnon, P. 1974. Termes à longue périodes dans le système solaire. Astron. Astrophys. 30, 141-154.

Brouwer, D., Van Woerkom, A.J.J. 1950. The secular variations of the orbital elements of the principal planets. Astron. Papers Am. Ephem. XIII, part II, 81107.

Busse, F.H. 1968. Steady fluid flow in a precessing spheroidal shell. J. Fluid Mech. 33, 739-751.

Cook, A.H. 1977. The moment of inertia of Mars and the existence of a core. Geophys. J. 51, 349-356.

Correia, A.C.M., Laskar, J., and Néron de Surgy, O. 2003. Long-term evolution of the spin of Venus I. Theory. Icarus 163, 1-23.

Costard, F., Forget, F., Mangold, N. and Peulvast, J.P. 2002. Formation of recent Martian debris flows by melting of near-surface ground ice at high obliquity. Science 295, 110-113.

Fanale, F.P. and Salvail, J.R. 1994. Quasi-periodic atmosphere-regolith-cap $\mathrm{CO}_{2}$ redistribution in the Martian past. Icarus 111, 305-316.

Folkner, W.M., Yoder, C.F., Yuan, D.N., Standish, E.M., and Preston, R.A. 1997. Interior structure and seasonal mass redistribution of Mars from radio tracking of Mars Pathfinder. Science 278, 1749-1751.

François, L.M., Walker, J.C.G., and Kuhn, W.R. 1990. A numerical simulation of climate changes during the obliquity cycle on Mars. J. Geophys. Res. 95, 1476114778 .

Gans, R.F. 1972. Viscosity of the Earth's core. J. Geophys. Res. 77, 360-366.

Goldreich, P. 1965. Inclination of satellite orbits about an oblate precessing planet. Astron. J. 70, 5-9.

Grimmett, G., Stirzaker, D. 2001. Probability and Random Processes. (Oxford Univ. Press, New York, 2001). 
Haberle, R. M., McKay, C. P., Schaeffer, J., Joshi, M., Cabrol, N. A., Grin, E. A 2000. Meteorological Control on the Formation of Martian Paleolakes. LPSC XXXI, Abstract \#1509.

Head, J.W. and Marchant, D.R. 2003. Cold-based Mountain Glaciers on Mars: Western Arsia Mons Fanshaped Deposits. LPSC XXXIV, Abstract \# 1247.

Imbrie, J., and Imbrie, K.P. 1979. Ice Ages: Solving the Mystery, Harvard University Press, MA

Jakosky, B.M. and Carr, M.H. 1985. Possible precipitation of ice at low latitudes of Mars during periods of high obliquity. Nature 315, 559-561.

Jakosky, B.M., Henderson, B.G. and Mellon, M.T. 1995. Chaotic obliquity and the nature of the Martian climate. J. Geophys. Res. 100, 1579-1584.

Kinoshita, H. 1977. Theory of the rotation of the rigid Earth. Cel. Mech. 15, 277-326.

Lambeck, K. 1979. On the orbital evolution of the Martian satellites. J. Geophys. Res. 84, 5651-5658.

Lambeck, K. 1980. The Earth's variable rotation. (Cambridge University Press, 1980).

Laskar, J. 1988. Secular evolution of the solar system over 10 million years. Astron. Astrophys. 198, 341362.

Laskar, J. 1990. The chaotic motion of the solar system. A numerical estimate of the size of the chaotic zones. Icarus 88, 266-291.

Laskar, J., Joutel, F., Boudin, F. 1993. Orbital, precessional, and insolation quantities for the Earth from -20 Myr to +10 Myr. Astron. Astrophys. 270, 522533.

Laskar, J., Robutel, P. 1993. The chaotic obliquity of the planets. Nature 361, 608-612.

Laskar, J. 1994. Large-scale chaos in the Solar System. Astron. Astrophys. 287, L9-L12.

Laskar, J. 1996. Large scale Chaos and marginal stability in the Solar System. Celest. Mech. \& Dyn. Astron. 64, 115-162.

Laskar, J. 1999a. The limits of Earth orbital calculations for geological time scale use. Phil. Trans. R. Soc. Lond. A. 357, 1735-1759.

Laskar, J. 134-150. : 1999b, Introduction to frequency map analysis, in Proc. of NATO ASI Hamiltonian Systems with Three or More Degrees of Freedom, C. Simò, ed. Kluwer.

Laskar, J., Robutel, P. 2001. High order symplectic integrators for perturbed Hamiltonian systems. Celest. Mech. 80, 39-62.
Laskar, J. 2001. Astronomical Solutions for Paleoclimates Studies. (AGU Fall Meeting 2001 Abstracts U11A-01, 2001).

Laskar, J., Levrard, B., and Mustard, J.F. 2002. Orbital forcing of the Martian polar layered deposits. Nature 419, 375-377.

Laskar, J., Robutel, P., Joutel, F., Gastineau, M., Correia, A., Levrard, B. 2004. A long term numerical solution for the insolation quantities of the Earth. (in preparation, 2004).

Laskar, J. 2004. A comment on 'Accurate spin axes and solar system dynamics: Climatic variations for the Earth and Mars'. Astron. Astrophys. 416, 799-800.

Lasota, A., and Mackey, M. C. 1994. Chaos, Fractals, and Noise. (Springer-Verlag, New York, 1994).

Leighton, R. B. and Murray, B. C. 1966. Behavior of carbon dioxide and other volatiles on Mars. Science 153, 136-144.

Levrard, B., Laskar, J. 2003. Climate friction and the Earth's obliquity. Geophys. J. Int. 154, 970-990.

Levrard, B., Forget, F., Laskar, J. and Montmessin, F. 2003. A GCM recent history of the Northern Martian polar layered deposits. Third International Conference on Mars Polar Science and Exploration Abstract\#8096, [CD-ROM].

Lumb, L.I., and Aldridge, K.D. 1991. On viscosity estimates for the Earth's fluid outer core-mantle coupling. J. Geophys. Geoelectr. 43, 93-110.

Mellon, M.T. and Jakosky, B.M. 1995. The distribution and behavior of Martian ground ice during past and present epochs. J. Geophys. Res. 100, 11781-11800.

Mignard, F. 1981. Evolution of the Martian satellites. Month. Not. Roy. Astron. Soc. 194, 365-379.

Mischna, M. A., Richardson, M. I., Wilson, R. J. and McCleese, D. J. 2003. On the orbital forcing of Martian water and $\mathrm{CO} 2$ cycles: A general circulation model study with simplified volatile schemes. J. Geophys. Res. 108 (E6), doi:10.1029/2003JE002051.

Murray, B. C., Ward, W. R., Yeung, S. C. 1973. Periodic Insolation Variations on Mars. Science 180, 638-640.

Mustard, J.F., Cooper C.D. and Rifkin, R.K. 2001. Evidence for recent climate change on Mars from the identification of youthful near-surface ground ice. Nature 412, 411-414.

Néron de Surgy, O., and Laskar, J. 1997. On the long term evolution of the spin of the Earth. Astron. Astrophys. 318, 975-989. 
Newhall, X. X., E. M. Standish, and J. G. Williams 1983. DE 102 - A numerically integrated ephemeris of the moon and planets spanning forty-four centuries.. Astron. Astrophys. 125, 150-167.

Pais, M.A., Le Mouël, J.L., Lambeck, K., and Poirier, J.P. 1999. Late Precambrian paradoxical glaciation and obliquity of the Earth - a discussion of dynamical constraints. Earth Planet. Sci. Lett. 174, 155-171.

Pijpers, F.P. 1998. Helioseismic determination of the solar gravitational quadrupole moment. M.N.R.A.S. 297, L76-L80.

Poincaré, H. 1910. Sur la précession des corps déformables. Bull. Astron. 27, 321-356.

Poirier, J.P. 1988. Transport properties of liquid metals and the viscosity of the Earth's core. Geophys. J. 92, 99-105.

Quinn, T. R., Tremaine, S., and Duncan, M. 1991. A three million year integration of the earth's orbit. Astron. J. 101, 2287-2305.

Richardson, M.I. and Wilson, R.J. 2000. Changes in the Martian Circulation and Climate in Response to Orbital Parameter Variations. Bull. Am. Astron. Soc. 32, 1092 .

Richardson, M.I. and Wilson, R.J. 2002. Investigation of the nature and stability of the Martian seasonal water cycle with a general circulation model. J. Geophys. Res. 107(E5), doi:10.1029/2001JE001536.

Roberts, P.H., and Stewartson, K. 1965. On the motion of a liquid in a spheroidal cavity of a precessing rigid body, II. Proc. Camb. Phil. Soc. 61, 279-288.

Rochester, M.G. 1976. The secular decrease of obliquity due to dissipative core-mantle coupling. Geophys. J.R.A.S. 46, 109-126.

Rubincam, D.P. 1990. Mars: change in axial tilt due to climate ?. Science 248, 720-721.

Rubincam, D.P. 1993. The obliquity of Mars and "climate friction". J. Geophys. Res. 98, $10827-10832$.

Saha P., Tremaine S. 1994. Long-term planetary integration with indivudual time steps. Astron. J. 108 (5), 1962-1969.

Standish 1998. JPL Planetary and Lunar Ephemerides, DE405/LE405. JPL-IOM , 312F-98-048.

Stewartson, K., and Roberts, P.H. 1963. On the motion of a liquid in a spheroidal cavity of a precessing rigid body. J. Fluid Mech. 33, 1-20.

Sussman, G. J. and J. Wisdom 1992. Chaotic Evolution of the Solar System. Science 257, 56-62.
Toon, O. B., J. B. Pollack, W. Ward, J. A. Burns, and K. Bilski 1980. The astronomical theory of climatic change on Mars. Icarus 44, 552-607.

Touma, J. and J. Wisdom 1993. The chaotic obliquity of Mars. Science 259, 1294-1297.

Varadi F., Bunnegar B., Ghil M. 2003. Successive refinements in long-term integrations of planetary orbits. Astrophysical J. 592, 620-630.

Ward, W. R. 1973. Large-scale variations in the obliquity of Mars. Science 181, 260-262.

Ward, W. R. 1974. Climatic Variations on Mars: I. Astronomical theory of insolation. J. Geophys. Res. 79, $3375-3386$.

Ward, W. R., Murray, B.C. and Malin, M.C. 1974. Climatic Variations on Mars: II. Evolution of Carbon Dioxide Atmosphere and Polar Caps. J. Geophys. Res. 79, 3387-3395

Ward, W. R. 1979. Present obliquity oscillations of Mars - Fourth-order accuracy in orbital E and I. J. Geophys. Res. 84, 237-241.

Ward, W. R., J. A. Burns, and O. B. Toon 1979. Past obliquity oscillations of Mars - The role of the Tharsis uplift. J. Geophys. Res. 84, 243-259.

Ward, W. R. and D. J. Rudy 1991. Resonant obliquity of Mars? Icarus $\mathbf{9 4}, 160-164$

Williams, J. G., D. H. Boggs, C. F. Yoder, J. T. Ratcliff, and J. O. Dickey 2001. Lunar rotational dissipation in solid body and molten core. J. Geophys. Res. 106, 27933-27968.

Wisdom, J., Holman, M. 1991. Symplectic Maps for the N-Body Problem. Astron. J. 102, 1528-1538.

Wisdom, J., Holman, M., Touma, J. 217-244. : 1996, Symplectic correctors, in Integration algorithms and classical mechanics (Toronto, ON, 1993), Fields Inst. Commun., 10, Amer. Math. Soc., Providence, RI.

Yoder, C. F. and E. M. Standish 1997. Martian precession and rotation from Viking lander range data. $J$. Geophys. Res. 102, 4065-4080.

Yoder, C.F., Konopliv, A.S., Yuan, D.N., Standish, E.M., and Folkner, W.M. 2003. Fluid core size of Mars from detection of the solar tide. Science 300, 299-303.

Zhang, C.Z. 1994. The Martian mean moment-of-inertia and the size of the Mars core. Earth, Moon, and Planets 64, 117-124. 